\title{
EDIFICI PER GLI DEI. EVERGETI PUBBLICI E PRIVATI NEI SANTUARI DEL PELOPONNESO DEL II SECOLO D.C.* \\ BUILDINGS FOR THE GODS. \\ Public AND PRIVATE BENEFACTORS \\ IN THE 2ND CENTURY AD PELOPONNESE SANCTUARIES
}

\author{
Annalisa lo Monaco \\ SAPIENZA UNIVERSITÀ DI ROMA \\ ANNALISA.LOMONACO@UNIROMA1.IT
}

\section{RIASSUNTO}

Quali edifici sono dedicati agli dei nel II secolo d.C.? Chi li pagava? Esiste una predilezione per un particolare genere di edifici (funzionali, di recezione, sacri in senso stretto) a seconda che si tratti di interventi finanziati dall' imperatore o da una committenza pubblica (i demi e i consigli locali), o che siano invece interventi privati? Questo articolo offre una rassegna delle principali tipologie di interventi edilizi e architettonici

\section{Abstract}

Which buildings are dedicated to the gods in the 2 nd century AD? Who paid for them? Is there a preference for a particular kind of buildings (functional, reception, sacred) depending on whether they are interventions financed by the emperor or a public commission (the demi and local councils), or whether it is instead a private intervention? This article offers an overview of the main types of build-

* Questo articolo è stato realizzato all' interno del progetto dell' Università Pablo de Olavide Adriano y la integración de la diversidad regional. Una perspectiva histórica e historiográfica (HAR201565451-c2-1 MINECO/FEDER). 
individuabili nel II secolo d.C. nei santuari del Peloponneso, esaminandoli in diacronia a seconda della loro funzione specifica (terme, teatri, edifici di assemblea) e mettendo in luce in che modo essi cambiarono la percezione e la vita quotidiana all' interno delle aree sacre. ing and architectural interventions that can be identified in the 2nd century AD. in the sanctuaries of the Peloponnesus, examining them in diachrony according to their specific function (thermal buildings, theaters, assembly buildings) and highlighting how they changed perception and daily life within the sacred areas.

\section{Parole chiave}

Peloponneso, santuari, evergeti, imperatori, dediche

\section{KeYWORDS}

Peloponnesus, sanctuaires, benefactors, imperators, dedications

Fecha de recepción: 20/12/2018

Fecha de aceptación: 18/03/2019 
Strade e PONTI, ACQUedotti, TERMe, teatri “Alla romana” e altro ancora. Nella Grecia di età imperiale si costruisce senza sosta. ${ }^{1}$ Gli interventi interessano sia i nuclei urbani sia alcuni dei più frequentati e antichi santuari. Fin dalla creazione della provincia Achaia, e poi con un incremento notevole nel II secolo d.C., nuovi modelli architettonici e modalità costruttive avevano iniziato a trasformare con le loro novità dirompenti paesaggi antropici di più antica (e altra) memoria. ${ }^{2}$ Sebbene con qualche variante locale dettata soprattutto dalla differente reperibilità di alcuni materiali, le grandi città iniziarono così ad avere finalmente un aspetto "romano". Una sempre crescente attenzione dedicata negli ultimi anni da un lato a tecniche edilizie, maestranze e cantieri attivi nella provincia Achaia $^{3}$ e dall'altro a singole tipologie di edifici pubblici (teatri, ${ }^{4}$ terme,${ }^{5}$ ninfei, ${ }^{6}$ acquedotti e infrastrutture ${ }^{7}$ ) e privati (mauso$\mathrm{lei}^{8}$ e domus ${ }^{9}$ ) consente finalmente di avere una visione di insieme ampia. Affiorano così nuove domande. In che misura tale fermento edilizio interessa anche i santuari? Giunge a modificarne lo skyline secolare? E ancora, che edifici erano dedicati agli dei nel II secolo d.C.? Chi li pagava? Esiste una predilezione per una particolare categoria (complessi funzionali, di recezione, sacri in senso stretto) a seconda che si tratti di in-

1. Stante la vastità della materia, si inseriscono di seguito le citazioni bibliografiche più recenti o attinenti ai temi trattati, rimandando a esse per la citazione della letteratura precedente.

2. Si pensi all' introduzione dell'opera cementizia con paramento, alle murature in incerto e reticolato con impiego di laterizio e pietra in luogo del tufo, all'utilizzo delle volte in laterizio con sovrastante conglomerato, e corsi orizzontali di laterizi su tutto lo spessore della muratura (Vitti, 2016a, pp. 68-69; Vitti, 2016b, pp. 194-195).

3. Vitti e Vitti, 2010; Vitti, 2016a e 2016b.

4. Nielsen, 2002; Bressan, 2009; Di Napoli, 2013.

5. Yegül, 1991 e 2015; Wassenhoven, 2012.

6. Longfellow, 2012; Rogers, 2018.

7. Si veda al riguardo il recentissimo Tassios, 2018, con bibl. prec.

8. Flämig, 2007; Dekoulakou, 2009.

9. Bonini, 2006. 
terventi finanziati dall' imperatore o da una committenza pubblica (i demi e i consigli locali) o che siano invece doni privati? Ci si muove ancora nel solco della tradizione ellenistica o si scelgono soluzioni aggiornate, più vicine ai nuovi bisogni? Segnalava Günther Schörner qualche anno fa come, nel computo complessivo delle dediche di votivi offerti agli dei nella Grecia di età romana, la dedica di edifici costituisca solo il $7 \%$ del totale: ${ }^{10}$ il dato è davvero esiguo se paragonato alle dediche di statue-ritratto (che si assestano intorno a un 27\%) e persino degli altari (al 22\%).

\section{GLI EDIFICI}

\subsection{NUOVI E VECCHI TEMPLI}

Nel secolo dei "buoni imperatori", così come era stato già in età ellenistica, ${ }_{11}^{11}$ gli interventi evergetici che riguardano erezione e dedica di nuovi edifici templari sono davvero esigui. ${ }^{12}$ Nel Peloponneso, a Epidauro si costruisce ex novo un edificio in età adrianea (un naòs completo di agalma dedicato a Telesforo da un altrimenti ignoto Fabullos, ${ }^{13} \mathrm{vd}$. tab. 2, cat. 3) e due in età antoniniana, entrambi finanziati dal senatore Antoninus (un luogo di culto sacro agli dei epidotai ${ }^{14}$ e un naòs dedicato a Hygiea, Asclepio e Apollo egiziani, ${ }^{15} \mathrm{vd}$. tab. 2, cat. 1). Forse a seguito di un terremoto ricordato in un' iscrizione si interviene anche a Isthmia, con un programma complesso, scandito in oltre un decennio e reso possibile dalla munificenza del senatore locale $\mathrm{P}$. Licinius Priscus Iuventianus ${ }^{16}$ (tab. 2, cat. 6-7). Si erigono così interi complessi, dotati di peribolo, statue di culto e arredi: gli altari degli dei patri con pronao, il naòs di Helios, i templi di Demetra, Kore, Dioniso e Artemide, e ancora i templi di Eueteria e Kore e il Ploutonion. La rifondazione dei sacra di Isthmia pare non risparmiare nessuno!

10. Schörner, 2013, pp. 198 e 211, fig. 2.

11. Schmidt-Dounas, 2000, pp. 14-15.

12. Gunther Schörner offre al riguardo un dato forse troppo minimale: "Almost no temples were dedicated" (Schörner, 2013, p. 199).

13. Da Milena Melfi identificato con l'edificio $\Omega$ nei pressi delle terme nord-orientali (Melfi, 2007a, p. 109 e tab. 7, n. 456; Melfi, 2007b, p. 252).

14. Milena Melfi intende l'Epidoteion citato da Pausania non come un edificio a sé stante, ma quale risistemazione del recinto delle arule (Melfi, 2007a, pp. 106-111; Melfi, 2007b, p. 248).

15. Non identificabile il naòs dedicato da Antoninus a Hygiea, Asclepio e Apollo egiziani (Melfi, 2007a, pp. 111-112).

16. Per la vasta bibliografia sul personaggio, rimando a Galli, 2001, pp. 57-62 e Torelli, 2010, p. 569 n. 19. 


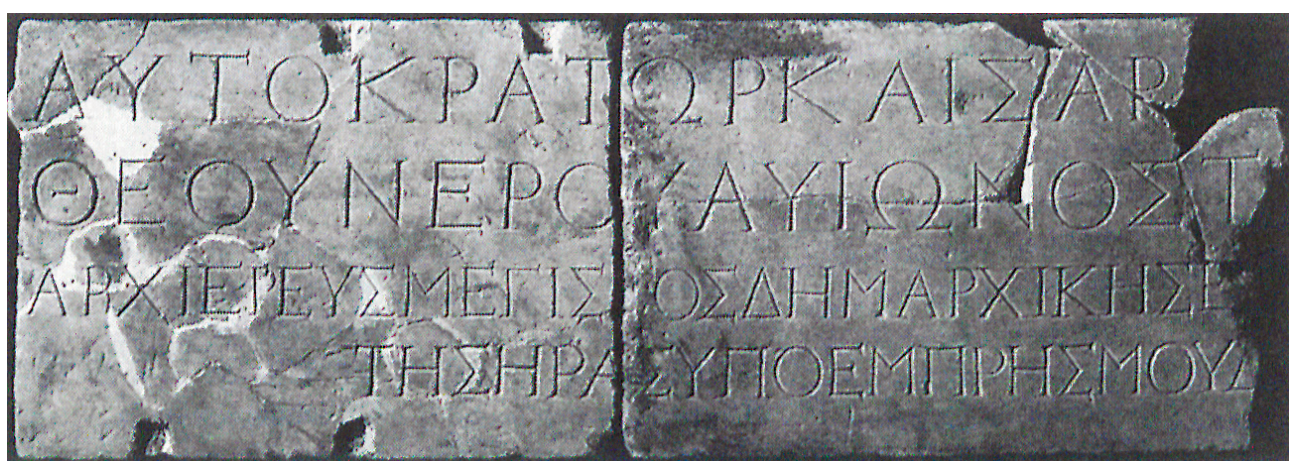

Fig. 1. Heraion presso Argo: iscrizione monumentale marmorea con indicazione dei restauri di Adriano (da Piérart, 1995)

Se in linea di massima si costruisce poco, si restaura però parecchio, e il dato non sorprende. ${ }^{17} \mathrm{Si}$ passa da interventi di restauro di vecchi edifici in stato ormai fatiscente a ricostruzioni vere e proprie, di grande impatto monumentale. Al santuario di Poseidon Hippios presso Mantinea (tab. 1, cat. 2), in cui Adriano giunge all' inizio del $125,{ }^{18}$ il progetto di ricostruzione fu complesso: una straordinaria gestione del cantiere, demandata a ispettori incaricati di sorvegliare i lavori e addirittura di impedire la visione del cantiere interno a curiosi e agli stessi operai (!), permise di inglobare il vecchio edificio ormai in rovina all' interno del nuovo, in modo da preservarlo senza distruggerlo. Non sappiamo come ci riuscirono, e quali furono le soluzioni adottate. A lavori ultimati, l'edificio si doveva comunque presentare in una veste del tutto nuova: in forme e materiali moderni, sintassi e linguaggio architettonico dovevano immediatamente denunciare, fin dall'esterno, l'aggiornamento al presente; all' interno la sacralità insita nel vecchio edificio era però salva, con un intervento di "tutela" che è una vera rarità nel mondo antico. Non conosciamo l'organizzazione dell'altro cantiere finanziato direttamente da Adriano, il restauro di un tempio di Hera (Antheia o Akraia?) presso Argo, distrutto da un incendio ${ }^{19}$ (tab. 1, cat. 1). L'intervento, non

17. Un utile inquadramento sulle teorie inerenti il restauro in età imperiale, con opportuna distinzione tra restauro come intervento manutentivo e restauro effettivo, è offerto da Calandra, 2007.

18. Lo Monaco, 2014, pp. 28-30, con bibl. prec.

19. I frammenti dell' iscrizione provengono da un pozzo nell'agorà e dagli scavi dell'orchestra del teatro. Essendo di peso non considerevole, non si può del tutto escludere che siano stati spostati anche considerevolmente dal luogo di collocazione originario. 
citato da Pausania, è noto grazie a un' iscrizione monumentale incisa su una lastra marmorea in eleganti lettere apicate (fig. 1), che restituiscono la titolatura di Adriano e consentono una datazione non posteriore al $128 .{ }^{20} \mathrm{I}$ lavori, finanziati nel corso della visita di Adriano del 124, furono dunque completati nel volgere di pochi anni. Le dimensioni delle lastre e il loro sviluppo complessivo, calcolato intorno ai 3.80 $\mathrm{m}$ di lunghezza, assicurano che l'iscrizione fosse apposta sull'architrave dell'edificio. È dunque anche in questo caso un intervento di rifacimento, più che di restauro integrativo: forse simile, nella concezione generale, a quello intrapreso dallo stesso Adriano pressoché contemporaneamente al tempio di Apollo a Megara, con la trasformazione integrale del vecchio edificio in mattoni in uno in marmo. ${ }^{21}$

Ancora diverso è l'intervento messo in atto negli stessi anni nel santuario di Poseidon a Isthmia ${ }^{22}$ (tab. 2, cat. 6). Qui, la necessità di una ristrutturazione a solo pochi anni dagli importanti lavori di età flavia deriva dalla volontà di inserire all' interno dello spazio racchiuso dal peribolo un tempietto dedicato a Palaimon: così, si allarga il peribolo a est, dotandolo anche di un propylon monumentale, e si aggiunge un piccolo basamento sormontato dalla statua del giovane eroe (fig. 2). Non si trasforma invece il sistema del sacrificio, che si avvale ancora dell' enagisterion (pozzo B) già inaugurato in età flavia. Qualche anno più tardi, nella cornice dei lavori finanziati da Iuventianus, si interviene ancora sul peribolo, spostandolo più a sud e dotandolo di un nuovo e monumentale ingresso. A mutare sono ancora una volta accesso e visuale di entrata al santuario, enfatizzata anche grazie alla nuova forma semicircolare dell'accesso al peribolo. Più radicale è l'intervento sul Palaimonion, spostato a sud e monumentalizzato nelle forme di una tholos coperta a cupola con statua, nell'occasione raccordata a un nuovo enagisterion (pozzo C) che modifica anche la cornice del sacrificio. Gli interventi avvengono forse sotto gli auspici del clero locale, come appare dal coinvolgimento certo nelle operazioni del mantis Blastos. ${ }^{23}$

20. Sull' iscrizione, vd. da ultimo Piérart, 1995.

21. Sull' intervento al tempio di Apollo a Megara, cf. Lo Monaco, 2014, p. 28, con bibl. prec.

22. Una revisione critica della bibliografia dell' intero complesso, per fasi, è offerta da ultimo da Mario Torelli (Torelli, 2010).

23. Per il ruolo di Blastos rimando all'analisi di Mario Torelli (Torelli, 2010, pp. 577-580).

ARYS, 16, 2018 [139-176] ISSN 1575-166x 


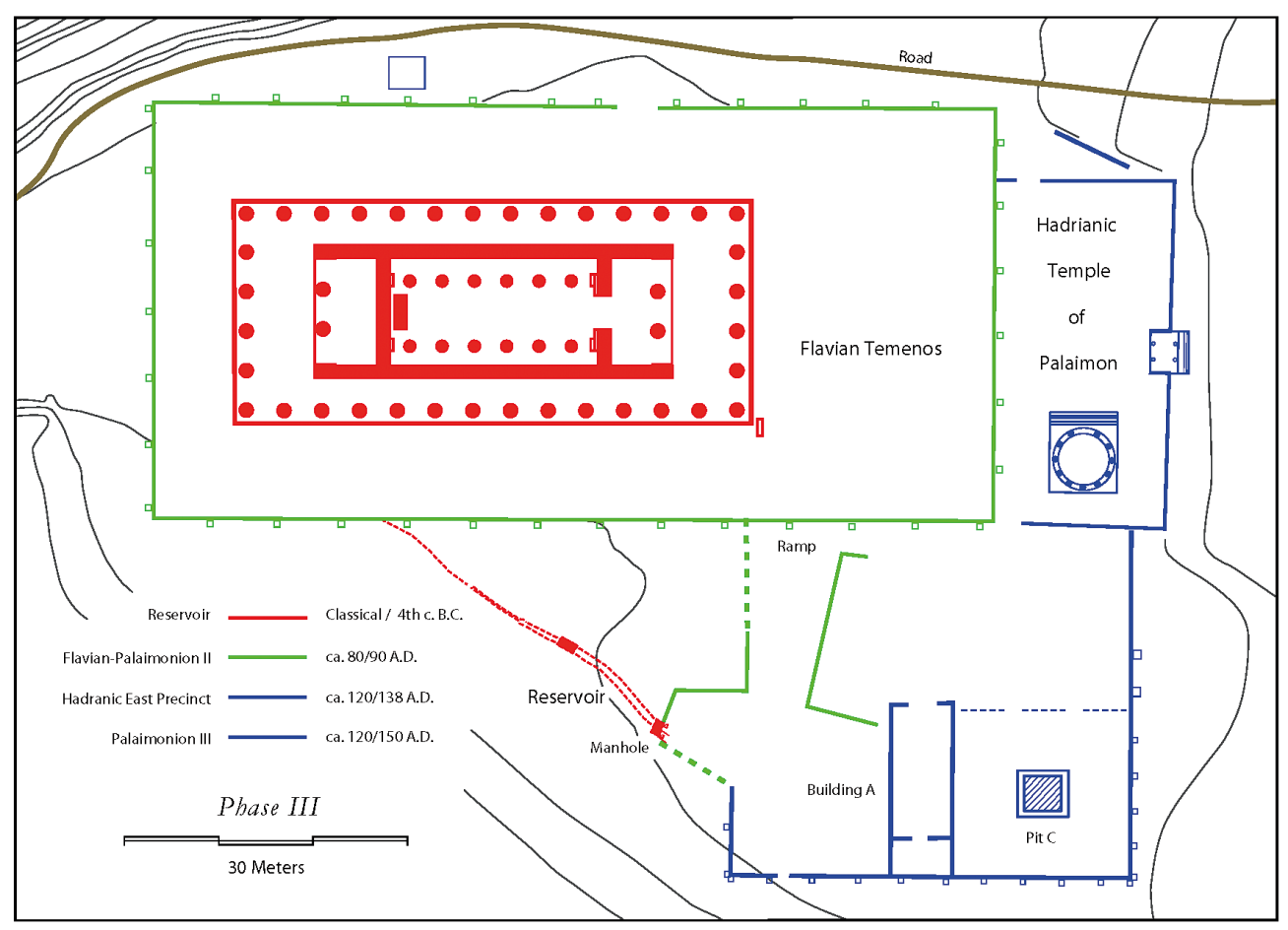

Fig. 2. Isthmia, santuario di Poseidon: pianta di fase degli interventi adrianei (da https://lucian.uchicago.edu/blogs/isthmia/reconstructions/southeast-precinct/phase-iib/ consultato in data 14/12/2018)

\subsection{NUOVI EDIFICI PER NUOVI SPETTACOLI}

Nel solco della tradizione ellenistica è il finanziamento di teatri e odeia. ${ }^{24}$ All' interno dei santuari si intraprendono sia ristrutturazioni (Isthmia, tab. 3, cat. 13) sia nuove erezioni (odeion di Epidauro, ${ }^{25}$ tab. 3, cat. 8). Gli interventi raggiungono lo scopo di ammodernare i vecchi edifici teatrali, rendendoli più vicini al gusto corrente e più occidentali, grazie a espedienti quali l'inclusione del velum o l'addizione di un basso

24. Barbara Schmidt-Dounas registra i casi del teatro di Tegea, dono di Antioco IV, e dei teatri di Delfi e Rodi, doni di Eumene II; l'unico odeion donato in età ellenistica è quello di Ariobarzane II ad Atene (Schmidt-Dounas, 2000, pp. 61-68).

25. Sull'edificio, si rimanda alla recentissima analisi di Paolo Vitti (Vitti, 2016a, pp. 171-174). 


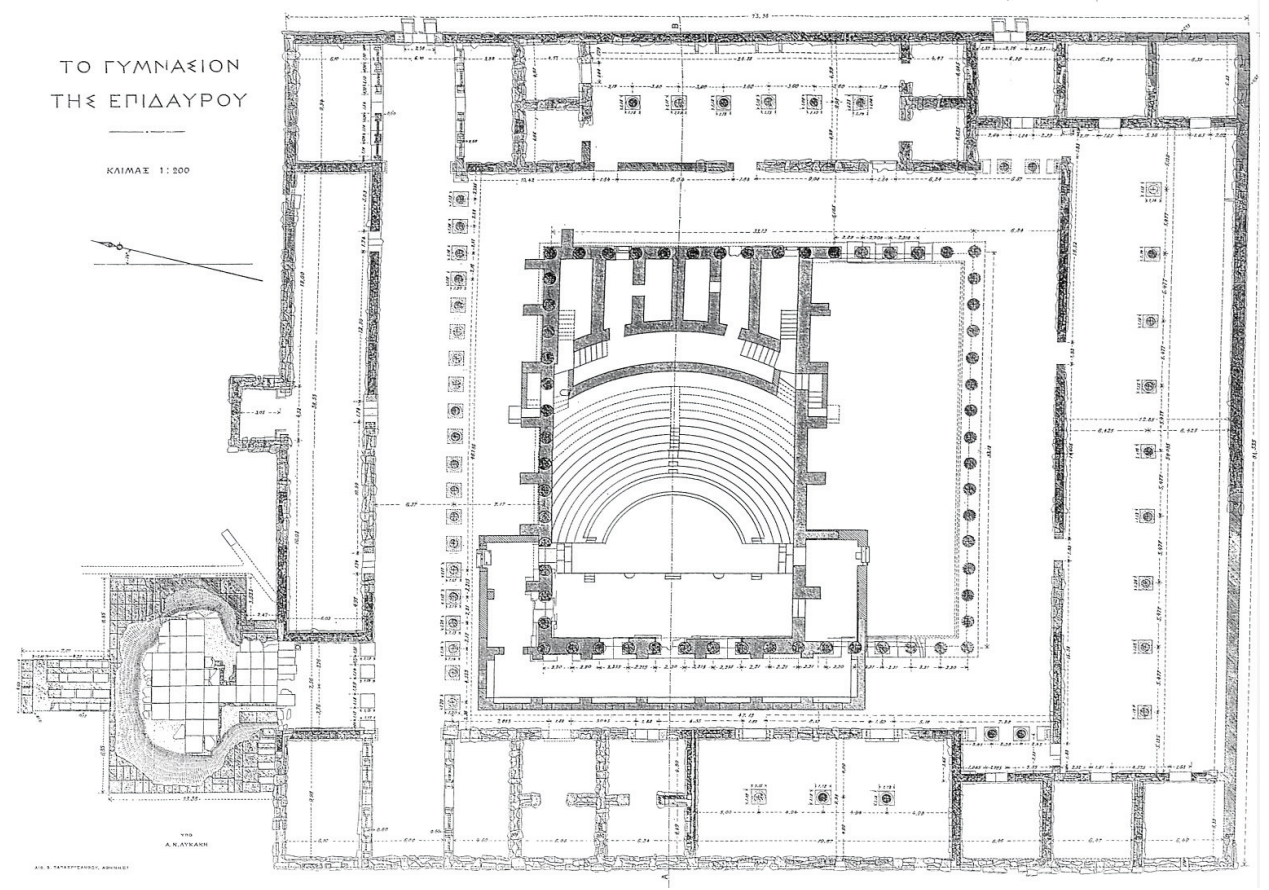

Fig. 3. Epidauro, santuario di Asclepio: odeion all' interno del cd.

"Ginnasio" (da Aslanidis, 1993)

e largo pulpitum al posto del più alto proscenio ellenistico. ${ }^{26}$ Tali trasformazioni si devono leggere in sincrono con le nuove attività che interessano gli edifici da spettacolo, utilizzati sempre più per performances musicali, oratorie e per cerimonie correlate al culto imperiale. A necessità liturgiche è da imputare verosimilmente la nuova edificazione a Epidauro dell' odeion, che, inserito all' interno del cd. ginnasio di età ellenistica almeno parzialmente in stato di abbandono, finirà per invaderne buona parte della corte del peristilio centrale (fig. 3 ). ${ }^{27}$ Cinque testi di componimenti poetici, inni

26. Di Napoli, 2010, passim.

27. Klimis Aslanidis ritiene che il ginnasio ellenistico fosse stato distrutto da un incendio e che proprio il suo stato di abbandono abbia consigliato l'erezione al suo interno dell' Odeion, per ragioni di risparmio (Aslanidis, 2003, pp. 301-302). 
liturgici e testi di agoni musicali furono inseriti nel nuovo edificio, evidentemente per essere qui intonati in determinati giorni festivi. ${ }^{28}$ L'odeion, capace di ospitare circa 1300 spettatori, si configura dunque come un vero e proprio "teatro sacro": è probabile che la sua erezione fosse suggerita da una riorganizzazione complessiva dell' intero ciclo liturgico. Assodata la funzione di luogo atto a performances canore sacre, va sottolineata la localizzazione all' interno del cd. ginnasio, scelta forse dettata dalla rapidità di esecuzione e da indubbi motivi di risparmio ${ }^{29}$ che portarono al riutilizzo integrale del vecchio stilobate e dei colonnati dorici del peristilio centrale (interamente nei tratti $\mathrm{N}$ e $\mathrm{W}$, parzialmente sul fronte $\mathrm{E}$ ). Se è vero che restarono in uso le ali $\mathrm{N}$ e $\mathrm{W}$ del cd. ginnasio, ${ }^{30}$ è altresì possibile che con tale localizzazione si mirasse anche a stabilire un nesso molto forte tra il nuovo odeion e la destinazione d' uso del vecchio complesso, adibito verosimilmente a banchetti rituali. Non solo: la copertura dell'edificio assicura che la nuova liturgia si svolgesse senza relazione alcuna (neanche visiva) con altri elementi del santuario, né edifici in elevato né scenari paesaggistici.

\subsection{L'ACQUA ALL'INTERNO DEI SANTUARI}

Sebbene la presenza di balnea nei santuari risalga già al V secolo a.C., la dedica evergetica di questi edifici è un fenomeno squisitamente romano, privo di precedenti nella tradizione ellenistica. ${ }^{31}$ Un recente intervento di Fikret K. Yegül mette in luce le molteplici funzioni di queste strutture: ${ }^{32}$ non solo igiene personale e cura del corpo, dopo lunghi e talora estenuanti viaggi, ma anche bagni cultuali, per immersione totale o parziale, bagni preliminari ai pasti cultuali, e, infine, bagni riservati agli atleti impegnati nelle gare. Bagni minerali termali in acque alcaline, funzionali alle guarigioni invocate, erano poi possibili all' interno dei santuari salutari meglio equipaggiati. I sanctuaries

28. Corpus di tutti gli inni: Wagman, 1995 e 2013, pp. 229-231. Vd. inoltre Galli, 2001, pp. 53-54 e 2004, pp. 338-343; Melfi, 2007a, pp. 24-125 e 2007b, p. 251. In un nuovo intervento dedicato a questi testi, R. Wagman chiarisce come le iscrizioni, lungi da essere già affisse in un edificio precedente e da questo traslate nel nuovo odeion, furono incise in questa fase in blocchi da costruzione reiempiegati e appositamente lisciati. L'intervento, tradizionalmente ascritto agli inizi del II secolo d.C., è abbassato cronologicamente a età antoniniana e riferito in via speculativa alla figura di Pythodorus (Wagman, 2013, pp. 220-222 e 227-228).

29. In tal senso, Aslanidis, 2003, pp. 301 e 303.

30. Aslanidis, 2003, p. 301.

31. Schörner, 2000, p. 314.

32. Yegül, 2015, p. 264; sul tema vd. anche Trümper, 2013, in part. pp. 52-62. 
baths sono dunque piuttosto diffusi in Grecia: ${ }^{33}$ nella sola Olimpia, in età imperiale avanzata, si contavano otto strutture di nuova erezione a ciò preposte ${ }^{34}$ e almeno tre sono riconoscibili nell' Asklepieion di Epidauro. ${ }^{35}$ Sebbene di norma situati all'esterno dell'area sacra cinta dal temenos, questi imponenti e sontuosi edifici, abbelliti con splendidi pavimenti marmorei policromi, eleganti tappeti musivi e ricchi apparati scultorei, dovevano finire per il mutare (decisamente in meglio!) la percezione complessiva e la vita stessa all' interno degli spazi sacri, ove finalmente era possibile rinfrescarsi, nuotare e prendersi cura del proprio corpo all' interno di sale di grande bellezza e magnificenza: Argo $^{36}$ (tab. 3, cat. 3-4), Delf, ${ }^{37}$ Eleusi ${ }^{38}$ Epidauro $^{39}$ (tab. 2, cat. 2), Isthmia ${ }^{40}$ (tab. 3, cat.

33. Terme annesse a santuari sono ben attestate anche nel mondo italico di età imperiale: cf. Delaine, 1999, p. 67 e passim.

34. Dislocate nei pressi di corsi d'acqua (Terme del Kladeos, Terme sud-west, non scavate; NordThermen), in prossimità degli edifici destinati al ricovero dei visitatori (vd. Ost-Thermen, SudThermen, Sud-West Termen, terme del Leonidaion o il complesso delle Kronion-Thermen a nord del pritaneo). Per comodità, si rimanda per la bibl. rel. a Wassenhoven, 2012, pp. 129-131, schede nn. 79-89 e Trümper, 2013, con fig. 17 a p. 59. Le terme sud-ovest, il cui impianto è datato da Fikret K. Yegül agli inizi del II sec. d.C. (Yegül, 2015, p. 256), risalgono secondo i dati di scavo al più tardi al I secolo d.C. e sembrerebbero da ritenere la sede di una corporazione di atleti (Sinn, Ladstätter e Martin, 1993, p. 156).

35. I complessi di nuova erezione in età imperiale sono: i bagni nei pressi della cisterna ellenistica, l'edificio $\mathrm{K}$ e le terme di Nord-Est, cui si può aggiungere l'intervento di ristrutturazione all' impianto greco, dotato in età imperiale di ipocausto (Melfi, 2007a, pp. 101-106; Trümper, 2014, p. 220). Nomina almeno sei complessi termali all' interno del santuario di Asclepio Paolo Vitti, senza però enumerarli (Vitti, 2016b, p. 171).

36. Vocazione termale potrebbe aver avuto ad Argo il complesso IX (denominato semplicemente "Roman Building" in Waldstein, 1902, pp. 134-136), a N-W del pianoro ove sorge il tempio; Galli, 2004, p. 347 suggerisce cautamente che si possa trattare della sede di un collegio, con sale riscaldate. Il complesso, accessibile grazie a un ampio atrio con colonnato centrale, presenta una serie di tre o quattro ambienti riscaldati, il cui funzionamento è possibile grazie a una fornace subito a ridosso, comunicanti con ambienti di dimensioni minori e funzione meno chiara. Nell'ottica dell'impianto termale, è curiosa l'assenza di sale adibite a frigidarium, a meno di non intendere come tale l'ambiente $\mathrm{O}$, definito da Waldstein cisterna o "tank for swimming". La presenza di un secondo piano, accertato grazie all' indicazione di più scale nel settore occidentale del complesso, sembrerebbe suggerire una funzione abitativa almeno per il piano nobile.

37. Il complesso degli "East Baths" a Delfi, di medie dimensioni, è eretto al di sotto della Stoà di Attalo, appena al di fuori dal peribolo.

38. Il complesso termale di Eleusi, immediatamente a ridosso dei grandi Propilei e risalente alla prima età severiana occupa un'estensione di circa 550 mq (Mylonas, 1961, p. 167, fig. 4, n. 40; Yegül, 2015, fig. 12.12). Poco oltre, è un secondo edificio termale, con bagni circolari (Mylonas, 1961, p. 167, fig. 4, n. 42).

39. Terme nord-orientali, i cui ambienti gravitano su una lunga sala centrale (B/A) coperta con volta a botte, che immette nei locali del calidarium e del frigidarium.

40. Il complesso termale di Isthmia, $100 \mathrm{~m}$ a NE rispetto al tempio di Poseidon, ma a quota sensibilmente inferiore, era facilmente raggiungibile dalla via che giungeva in salita dal teatro. Cf. Yegül, 2015. 


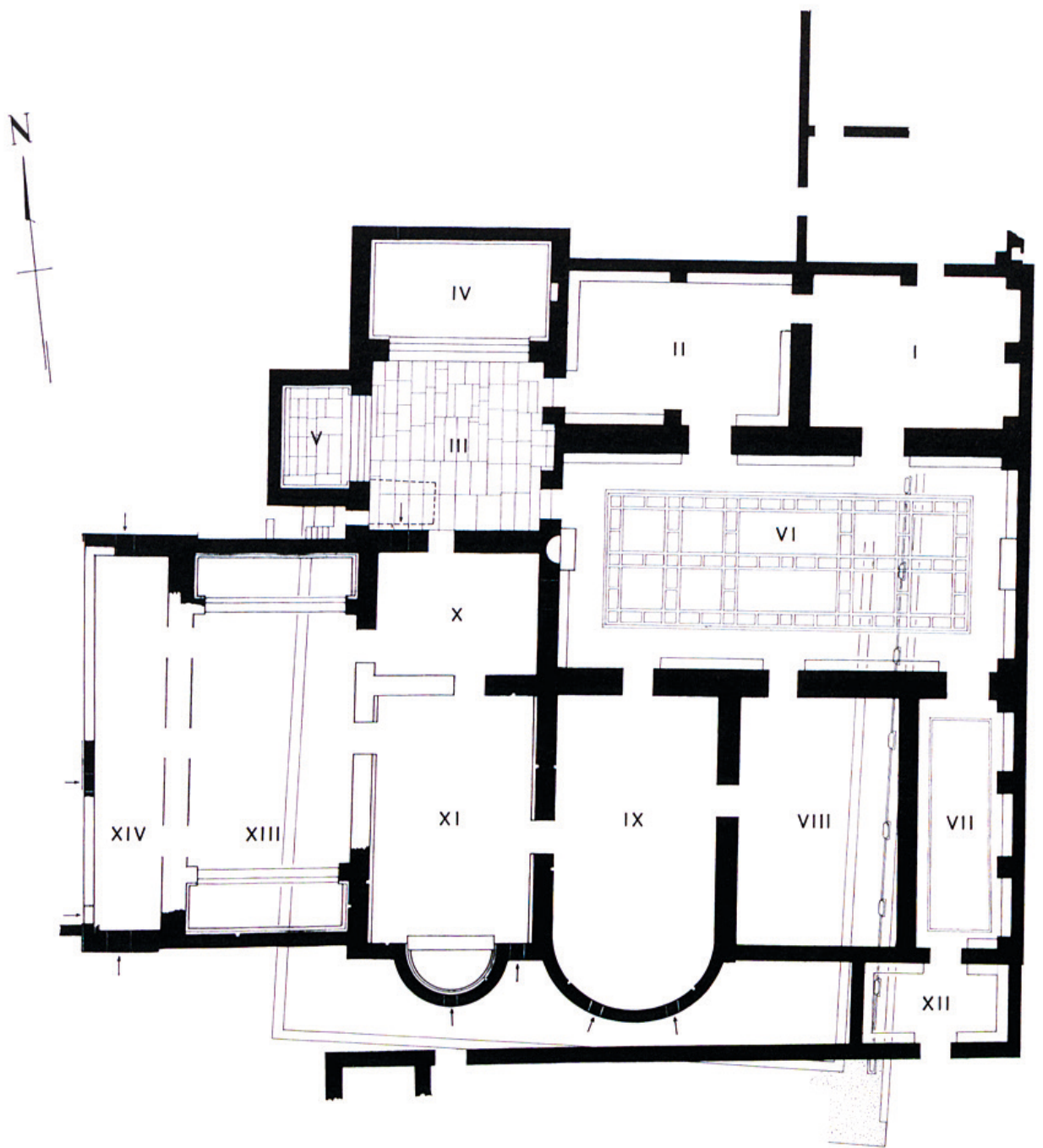

Fig. 4. Santuario di Poseidon a Isthmia, complesso delle grandi terme (da Yegül, 2015) 
14), Olimpia, Oropos ${ }^{41}$ ne sono una testimonianza concreta. ${ }^{42} \mathrm{Si}$ spiega così il frequente ricorso di alcuni tra i più facoltosi evergeti del periodo a donazioni di queste enormi (e costosissime) strutture. Il senatore Sextus Iulius Maior Antoninus Pythodorus (tab. 2, cat. 2), esplicitamente onorato dalla città di Epidauro come evergetes ${ }^{43}$ finanzia nel santuario tra le altre cose anche un grandioso impianto termale noto in antico con il

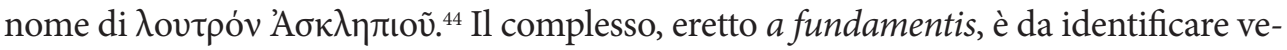
rosimilmente con le Terme nord-orientali, ${ }^{45}$ erette forse ancora all' interno del peribolo in un'area da sempre legata a rituali inerenti alla purificazione, e di immediato accesso dall'area più interna del santuario. A Ishtmia (tab. 3, cat. 14, fig. 4) era stato eretto da qualche decennio un complesso senza uguali (estensione di poco inferiore ai 2000 mq!), le cui sale non sono distribuite simmetricamente bensì secondo il principio della "hall type", ${ }^{6}$ con ambienti, a gruppi funzionali (sale riscaldate, frigidaria, apodyteria), distribuiti intorno e accessibili da una sala centrale, di norma rettangolare e di grandi dimensioni, secondo un percorso circolare che permetteva un movimento a rotazione completa in senso orario dal caldo al freddo.

La straordinaria novità che questi impianti recano con sé è proprio nella creazione di percorsi che, avvalendosi di nuove tecnologie, rendessero possibili (e molto gradevoli) permanenze più o meno prolungate in ambienti riscaldati prima e in sale fredde poi, secondo una concezione del tutto estranea al mondo tardo-classico e primo ellenistico dei balnea greci. Tale dirompente innovazione era poi segnalata anche a livello percettivo, grazie alla presenza delle grandiose coperture a volta, che sormontavano le più grandi di queste sale e che dovevano essere ben visibili anche da lontano. Le volte dovevano essere insomma una sorta di marker tridimensionale di una nuova realtà, in tutto romana ma ottimamente integrata persino negli spazi sacri. Fa bene Monika Trümper a sottolineare con energia, molto di recente, come l' installazione di questi impianti non fosse in relazione alcuna con lo svolgimento di

41. Yegül, 2015, pp. 260-261.

42. Differente il caso di Samo, ove una casa a peristilio adiacente al "tempio prostilo 2", di età antoniniana, è verosimilmente da intendere non come edificio termale ma quale sede di un collegio, rivolto a un gruppo ristretto di persone e collegato all'edificio cultuale (idea già in Galli, 2004, p. 346).

43. IG IV 684 ; Melfi, 2007a, p. 99 e tav. 7, n. 507.

44. Paus., II 27, 6; Schörner, 2000, pp. 309-310.

45. Concordo pienamente con la lettura già avanzata da Milena Melfi, che, scartata la possibilità di

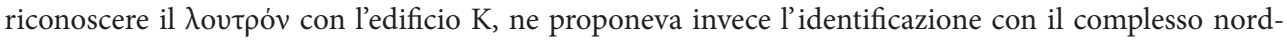
orientale (Melfi, 2007a, pp. 101-106).

46. Yegül, 2015, p. 247 (con datazione ai decenni centrali del II secolo d.C.), e 250 e nota 7 ("hall type building"), con bibl. prec. 
pratiche curative, neanche all' interno di santuari salutari: ${ }^{47}$ si tratta, in altre parole, del diffondersi di un costume sociale inteso in senso più ampio, di un nuovo modo di sentire e di vivere la quotidianità di spazi di secolare tradizione. Altra differenza di cui tener conto è che questi impianti, per loro stessa natura, presuppongono una frequentazione non regolamentata a livello corale, come di prassi negli spazi dei santuari, ma scandita da tempi personali e singoli: i visitatori dovevano essere liberi di entrare a proprio piacimento e di godere della successione di temperatura e acque, immersi per qualche ora in queste sale lussuose. L'impressione che se ne ricavava doveva essere duratura. Così, non è un caso che sia questo uno degli interventi prediletti dai grandi evergeti del tempo, e che non siano affatto registrate donazioni di imperatori o interventi di finanze pubbliche a favore di impianti termali. ${ }^{48}$ Pressoché contemporaneamente interventi evergetici molto simili sono rivolti in Asia Minore alla costruzione ex novo di impianti in scala monumentale, la cui capacità recettiva è stata messa in relazione all'enorme afflusso di pubblico in occasione delle grandi festività. ${ }^{49}$

Il funzionamento di tali edifici abbisognava come è ovvio di un grande quantitativo d'acqua, e la soluzione di tale problema all' interno di spazi sacri di più antica memoria non fu mai scontata. ${ }^{50}$ Così, è proprio la recentissima adduzione di acquedotti ad Argo, Epidauro e Corinto, ${ }^{51}$ tra gli altri, a rendere possibile la gestione idraulica delle terme di Argo, ${ }^{52}$ del loutron Asklepiou a Epidauro e delle grandiose terme di Pythodorus a Isthmia. ${ }^{53}$

47. Trümper, 2014.

48. Al conteggio complessivo va aggiunta la costruzione o il restauro a Tegea di un grande complesso, fornito almeno di un balaneion e una stoà, e dedicato da una Valeria S..., a spese proprie e verosimilmente del marito, ad Adriano Sebastòs, Olympios, Pythios e Panhellenios (IG V,2 127; Schörner, 2000, p. 309; RomPel I, 148-149, cat. ARC 166). Per le terme in territorio italico, raramente finanziate da denario pubblico in età medio-imperiale, cf. Delaine, 1999, p. 70.

49. Barresi, 2007, p. 138 e passim. A differenza delle terme-ginnasio micro-asiatiche, nelle terme peloponnesiache non risulta inserita alcuna Kaisersaal, sala decorata da edicole e statue, aperta su uno dei portici e spesso riservata alle manifestazioni del culto imperiale.

50. Facilitazioni per acqua sono già nella legge di Andania: Camia, 2011, p. 45. Si pensi che a Olimpia il problema fu definitivamente risolto solo la costruzione dell'acquedotto di Erode Attico dall'Alfeo all'Altis, per cui vd. infra.

51. Sull'acquedotto che captava le acque del lago Stinfalo cf. Vitti, 2016b, pp. 194-202 e da ultimo Lolos, 2018, pp. 98-108.

52. Il complesso, denominato "Bath A", è stato recentemente oggetto di un approfondito studio analitico da parte di P. Vitti, al cui lavoro si rimanda (Vitti, 2016a, pp. 85-108). Non del tutto accettata è la sua identificazione con un edificio sacro a Serapide o Asclepio. A favore di un Asklepieion Riethmüller, 2005, pp. 81-83.

53. Per la bibl. specifica sui singoli acquedotti, tutti di età adrianea, si rimanda a Vitti, 2016a, p. 73. 
Acqua che rinfresca, acqua nella quale bagnarsi, ma anche acqua che scorre a profusione, per il diletto di vista e udito. Lo spazio circostante ne risulta plasmato e fortemente condizionato. ${ }^{54}$ Non è un caso che, quasi simultaneamente, esplodano le dediche di ninfei e fontane monumentali, che, con i loro sontuosi apparati scultorei, magnificano gesta e memoria dei generosi committenti. Si va dalle fontane vicino alle terme nord-orientali a Epidauro, ${ }^{55}$ fino a impianti come il ninfeo di Argo con statua in nudità eroica di Adriano inserita nella nicchia di fondo, ${ }^{56} \mathrm{o}$ al sontuoso ninfeo di Olimpia, finanziato da Erode Attico ma dedicato nel nome della moglie, a due passi dal tempio di Hera (tab. 2, cat. 12).

\subsection{STRUtTURE RECETTIVE}

Solo episodica è l'attenzione riservata alla dedica di edifici recettivi, atti a ospitare o riparare stranieri o pellegrini nei santuari a più alta frequentazione. Al di là dei pur numerosi apprestamenti effimeri, ${ }^{57}$ l'esorbitante afflusso di turisti, pellegrini e curiosi dovette consigliare la realizzazione di impianti appositi. Così, non è un caso se due dei più facoltosi evergeti del periodo, i senatori Antoninus e Iuventianus, si occupino uno del restauro del portico di Kotys e forse del katagogion a Epidauro ${ }^{58}$ (tab. 2, cat. 1), l'altro di una stoà denominata Reguliana a Isthmia ${ }^{59}$ (tab. 2, cat. 7). Quest' ultimo intervento, forse possibile grazie alla carica di agoranomos rivestita da Iuventianus, ${ }^{60}$ si pone più come una ristrutturazione che come un restauro puro e semplice, se è vero che scopo recondito dell'operazione fosse quello di realizzare all' interno della struttura ben 50 oikoi nei quali alloggiare atleti in gara. Sempre a Epidauro, ma sul Kynortion, il santuario di Apollo Maleatas ospita una struttura, la cd. skanà, che, al-

54. Brenda Longfellow sottolineava come non si conoscano in Grecia acquedotti preesistenti all'età adrianea, e che il loro inserimento abbia comportato sensibili alterazioni dei paesaggi (Longfellow, 2012, pp. 134-135).

55. Melfi, 2007a, p. 125, con rif. a Epidauro 1999, pp. 49-50.

56. Sul ninfeo di Argo, vd. Longfellow, 2009, pp. 216-224 e da ultimo Vitti, 2016a, pp. 71-72 e fig. 2.3 (restituzione grafica della facciata), e 127-132, figg. 3.49-51.

57. Si vd. Elian., Var. Hist. 4, 9; Goldstein, 1978, pp. 8-100, con analisi dei casi di Delfi, Isthmia, Olimpia, Samo, Atene, Sparta, Andania, Elateia, Kos, Ionia, Karia e Licia.

58. Melfi, 2007a, pp. 115-116. Gli interventi al katagogion sono genericamente datati al II secolo. A differenza della ristrutturazione imperiale al Leonidaion di Olimpia, di recente a ragione riferita a età flavia (Specht, 2004), i lavori al katagogion non comportarono una radicale trasformazione interna degli ambienti, con la sola eccezione del porticato sud-orientale, il cui peristilio si trovò invaso dal prolungamento di setti murari di alcuni ambienti che su di esso si affacciavano (Kraynak, 1991).

59. Torelli, 2010, pp. 571-572.

60. Camia, 2011, p. 44. 
meno nella sua fase di impianto doveva forse avere una connotazione residenziale. ${ }^{61}$ A Olimpia, nel settore immediatamente a sud delle terme del Kladeos, ${ }^{62}$ si installano tra la metà del II e la metà del III sec. d.C. due complessi caratterizzati da vani disposti intorno a un peristilio con vasca centrale (tab. 3, cat. 17): l'apertura non assiale sembra denunciare per alcuni degli ambienti una funzione correlata allo svolgimento di pasti collettivi su klinai, laddove un numero più ridotto di vani potrebbe avere avuto una funzione più tradizionalmente residenziale. ${ }^{63}$

\subsection{UNA BIBLIOTECA, COMPLETA DI LIBRI}

Caso eccezionale, nel Peloponneso, è la dedica di una biblioteca, offerta nel II secolo d.C. da un Gaio Roufos nel santuario di Epidauro congiuntamente ad Apollo Maleatas e Asclepio ${ }^{64}$ (tab. 2, cat. 4). L'ignoto personaggio, originario di Napoli stante l' integrazione di Werner Peek, ${ }^{65}$ dedica agli dei guaritori una biblioteca, evidentemente l'edificio, forse insieme a tutto l'apparato librario ${ }^{66}$ finanziando il contestuale svolgimento di agoni. ${ }^{67} \mathrm{~A}$ differenza di quanto accertato a Pergamo, ${ }^{68}$ a Epidauro l'edificio in sé non è stato rinvenuto, né all' Asklepieion basso né sul Kynortion. Il rinvenimento dell' iscrizione, su una stele in marmo ora in frammenti, all' interno dell'edificio $K$ indusse Panagiotis Kavvadias a intendere un'ala della struttura come biblioteca, ma

61. Marco Galli (Galli, 2004, pp. 333-334) suggerisce l'idea che l'edificio fosse la sede di un collegio religioso; Milena Melfi, pur ritenendo la soluzione suggestiva per le fasi più recenti, per la fase di impianto preferisce pensare a un edificio a vocazione residenziale (Melfi, 2007a, p. 118).

62. L'area occidentale esterna all'Altis compresa tra l'angolo sud-occidentale della Palestra e il Kladeos aveva assunto, già a partire da età ellenistica, una fisionomia peculiare: è l'area del training e del ristoro, funzionale allo svolgimento di esercizi fisici, rigeneranti bagni, attività culturali e pernottamenti, anche all' interno di tende e apprestamenti effimeri (al riguardo cf. Lo Monaco, 2013).

63. OlBer VI 33-37, tav. 6 e 7.

64. Burzachechi, 1984, pp. 334-335, n. 8; Perilli, 2006, p. 491; Melfi, 2007a, pp. 123-124 e 2007b, p. 251.

65. Peek, 1969, n. 178.

66. L'iscrizione, assai frammentaria, è purtroppo molto integrata e la menzione dei libri ricade in lacuna.

67. Roberto Nicolai avanzava l'idea che si trattasse di agoni indetti per festeggiare la costruzione dell'edificio (Nicolai, 1988).

68. L'unica biblioteca rinvenuta archeologicamente è quella donata da Flavia Melitine a Pergamo, all' interno di una sala del santuario di Asclepio (I.Perg. III 38). La biblioteca di Gaio Stertinio Senofonte a Kos è nota invece solo su base epigrafica: la provenienza dell' iscrizione dalla I terrazza del santuario ha fatto ipotizzare che l'edificio fosse ivi allocato (AA 4 1903, 193-194; Burzachechi, 1984, pp. 309-311, n. 2; Interdonato, 2013, p. 63). 
tale lettura è lungi dall'essere accettata. ${ }^{69}$ Una raccolta di libri consultabili implica fruitori, meglio se colti: a chi dunque erano destinati i libri di Asclepio a Epidauro? Qui le opinioni divergono: c'è chi pensa a una raccolta specialistica, finalizzata a una ristrettissima consultazione colta e forse all' insegnamento, chi invece preferisce pensare a biblioteche atte a soddisfare le esigenze dei visitatori e lo svago dei pazienti, ${ }^{70} \mathrm{e}$ chi infine pensa a necessità inerenti terapie mediche e pratiche di guarigione. ${ }^{71}$

In verità, l'attestazione del dono di biblioteche nella prima età imperiale nei tre principali santuari di Asclepio del mondo antico (Pergamo, Kos ed Epidauro) farebbe pensare alla necessità comune di un aggiornamento del patrimonio librario a disposizione, forse per l'insorgere di nuove esigenze connesse allo sviluppo delle pratiche mediche. In ogni modo, ben presto gli Asklepieia maggiori si trasformarono in veri e propri centri di aggregazione culturale. ${ }^{72}$

\section{I COMMITTENTI}

\subsection{GLI IMPERATORI E LA CASA IMPERIALE}

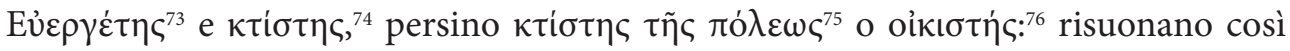
i meriti di Traiano, Adriano e Antonino Pio in basi di statue o altari dedicati a loro nome in numerose località dell' Achaia. All'appello manca curiosamente Marco Aurelio, per il quale non è attestato alcun epiteto che, dietro la consueta enfasi, possa adombrare qualche merito specifico in relazione ad attività edilizie. Tale profusione di ringraziamenti, talora ricorrenti persino in coppie e attestate in poleis e santuari distribuiti organicamente su tutta la provincia, solo in uno sparuto numero di casi

69. Kavvadias, 1900, pp. 157-158, seguito da Wagman, 2013, p. 223 n. 14. Critica sulla lettura di Kavvadias Milena Melfi, sulla base delle caratteristiche del locale, non congrue alle necessità di una biblioteca (Melfi, 2007a, p. 123). La dedica congiunta ad Apollo Maleatas fa preferire ad Ada Caruso una localizzazione della biblioteca nel santuario del Kynortion (Caruso, 2014, p. 75).

70. Guarducci, 1978, p. 211; Burzachechi, 1984, p. 335; Melfi, 2007a, p. 124.

71. Nicolai, 1988, in part. 37; Caruso, 2014, p. 68.

72. In tal senso, già Nicolai, 1988, p. 37.

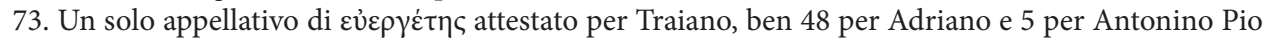

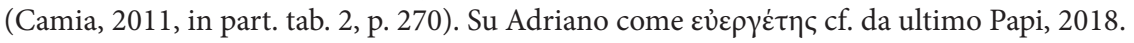

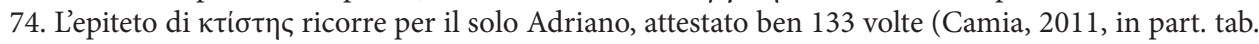
2, p. 271).

75. È il caso della dedica in favore di Antonino Pio a Tegea: IG V 2132 (1) (Camia, 2011, in part. tab. 2, p. 272).

76. Attestato nel solo caso di Adriano ad Atene (IG II-III ${ }^{2}$ 3306) ed Epidauro ( $I G \mathrm{IV}^{2}$ 606) (Camia, 2011, in part. tab. 2, p. 272). 
(Megara ed Epidauro) proviene da centri oggetto di effettive presenze fisiche in Achaia dell' imperatore in carica. ${ }^{77}$ Le visite imperiali sono in effetti non molto frequenti. Sono accertate visite dirette di Traiano ad Atene, ${ }^{78}$ di Adriano ad Atene, Eleusi, Megara, Epidauro, Argo, Mantinea, Abe e Delf, ${ }^{79}$ di Marco Aurelio (in compagnia del fratello Lucio Vero e del figlio Commodo) nelle sole Atene ed Eleusi. ${ }^{80}$ Antonino Pio in Grecia non metterà mai piede. ${ }^{81}$ Si tratta dunque per lo più di ringraziamenti in assenza, nel quadro di un modo ormai formulare, ben rodato e convenzionale di rivolgersi all' imperatore in carica. Persino nel caso di eventi calamitosi un loro intervento diretto è pressoché nullo, ${ }^{82}$ a fronte di una cospicua casistica di finanziamenti dagli imperatori in carica nel corso del I secolo d.C. a seguito di catastrofi naturali di grave entità. ${ }^{83}$ Lo stato di emergenza (terremoti certi nel II secolo almeno a Sicione, ${ }^{84}$ Lindos e Corinto) si affronta piuttosto grazie al contributo di facoltosissimi evergeti

77. Le sole Atene (IG II-III ${ }^{2}$ 3306), Eleusi (Clinton, 2005, n. 446), Epidauro (IG IV² 606) e Megara nel caso di Adriano (IG VII 70-72, 3491 (Camia, 2011, in part. tab. 2, pp. 270-272).

78. Fine del 113 d.C.: cf. Cass. Dio, LXVIII 17, 2; Halfmann, 1986, p. 184.

79. Per una più approfondita analisi delle iniziative di Adriano a favore di questi centri in occasione della sua visita e delle singole risposte delle comunità locali si rimanda a Lo Monaco, 2014. Su Atene, cf. da ultimo Luberto, 2018. Ho espunto dal novero dei centri oggetto di visita dell' imperatore quei casi in cui le testimonianze lascino intravedere una semplice manifestazione di lealtà all' imperatore, nel quadro della convenzionale adesione al potere centrale: pertanto, ho eliminato i casi di Trezene, Hermione, Lyosoura, Lebadea e il Trophonion; non verificabili risultano anche le visite di Adriano a Olimpia e Corinto (su questi due centri, cf. Birley, 1997, pp. 181-182; su Olimpia St. Specht in Sinn, Ladstätter e Martin, 1996, pp. 201-211; nella carta elaborata da Giacomo Fadelli in occasione della recentissima mostra ad Atene entrambe le tappe sono considerate come effettive: Fadelli, 2018, p. 33).

80. Sulle iniziazioni di Marco Aurelio, Lucio Vero e Commodo, cf. Clinton, 1989, pp. 1529-1534. Sui viaggi ad Atene ed Eleusi, cf. Halfmann, 1986, pp. 210-211 (Lucio Vero), 213 (Marco Aurelio).

81. Longfellow, 2012, p. 135.

82. Vd. l'intervento di Adriano a Cizico dopo il terremoto del 120/128 d.C. (Joh. Mal., 279); di Traiano a Thelpousa in Arcadia (SEG XI 1124, del 102/116); di Antonino Pio per il terremoto del 142/144 in parte della Licia Caria, a Kos e a Rodi (Paus., VIII 43, 4 e Malacrino, 2007, pp. 263-265); di Marco Aurelio a Smirne intorno al 178 d.C. (Cass. Dio, LXXI 32, 3); di Commodo a Nicomedia di Bitinia (Joh. Mal., 289).

83. Interventi di Augusto a Kos (dopo il terremoto del 26 a.C.: IvO 53, 1l. 7-11; Malacrino, 2007, p. 259); a Cipro (terremoto del 17 a.C.: Cass. Dio, LIV 23, 7); di Tiberio a favore delle città d'Asia colpite dal terremoto del 17 d.C. (fonti in Guidoboni, 1989, pp. 657-659); di Caligola ad Antiochia (terremoto del 37 d.C.: Joh. Mal., 243); di Claudio a Samo (terremoto del 47 circa: IG XII 6,1 483) e Creta (terremoto del 53: Joh. Mal., 250); Vitellio a Nicomedia di Bitinia (terremoto del 69: Joh. Mal., 259); Vespasiano a molte città colpite da terremoti o incendi, tra cui Corinto (Suet., Vesp. 17; Joh. Mal., 261); di Nerva a Diocesarea in Cilicia che viene per l'occasione rinominata Anazarbo (terremoto del 97: Joh. Mal., 267). Cf. Quaß, 1993, pp. 224-225.

84. Paus., II 7, 1. 
privati, quali Tiberio Claudio Antipatro a Lindos ${ }^{85}$ autore insieme al figlio della ricostruzione del santuario di Asclepio, e di P. Licinius Priscus Iuventianus a Corinto (tab. 2, n. 6). Sebbene non siano del tutto assenti lettere indirizzate alle poleis o alle comunità locali, ${ }^{86}$ in verità i buoni imperatori sono piuttosto lontani, forse distratti dalle guerre nordiche e orientali, e certamente poco presenti nella vita economica delle comunità locali dell' Achaia. Il loro favore si esplica, al più, nella concessione del mantenimento dei privilegi o nell'esenzione dal regime di tassazione vigente, come nei casi di Mothone (Traiano) e Pallantion (Antonino Pio), sulla scia delle concessioni, ancora di età repubblicana, a Epidauro, Delfi e forse a Elis. ${ }^{87}$

Unica eccezione è costituita da Adriano, il solo che, come detto, si incarica del restauro di alcuni prestigiosi edifici sacri, ormai diruti.

\subsection{GLI EVERGETI LOCALI}

Tra i regni di Traiano ed Adriano si registrano unicamente le donazioni di Fabullos a Epidauro (tab. 2, cat. 3), di A(ulus) Maecius Phaedrus e di C. Iulius Eurykles Herclanus L. Vibullius Pius a Mantinea (tab. 2, cat. 10-11) e di Poplios Memmios Agathokles a Tegea (tab. 2, cat. 13). Atti fortemente differenziati e non riconducibili a minimi comuni denominatori, né per il livello sociale dei donatori, né per la natura degli edifici dedicati e i loro significati intrinseci.

Di Fabullos non sappiamo nulla: ${ }^{88}$ l'assenza del gentilizio mostra che il personaggio non aveva la cittadinanza romana. ${ }^{89} \mathrm{Il}$ nome, un unicum in Grecia, è modellato sul cognomen latino Fabullus: ${ }^{90}$ potrebbe trattarsi di un personaggio di condizione

85. Lindos II, 449, ll. 13-16.

86. Oliver, 1989: vd. in particolare le lettere imperiali indirizzate a Astypaleia, Atene, Beroia, Coronea, Delfi, Gythion, Isthmia e Sparta. Per il Peloponneso sono attestate in verità le sole lettere di Traiano al sinodo di Isthmia (99 d.C., ibid., 139-140 n. 47) e di Adriano alla lega Achea (126 d.C., ibid., 195-200 n. 78 A-B), a Gythion (ibid., 228-229 n. 90) e a Sparta (ibid., 229-232 n. 91).

87. Mothone (Paus., IV 35, 3) e Pallantion (Paus., VIII 43, 1) sono dichiarate civitates liberae et immunes. Per Epidauro, Delfi e Elide cf. Zoumbaki, 2010, p. 115, n. 30.

88. Il personaggio non è altrimenti conosciuto, né il nome risulta mai attestato, in questa forma, nelle iscrizioni greche.

89. Cf. Rizakis, 1996. In linea di principio non si può escludere che il nostro Fabullus, di origine non italica, sfoggi un nudus nomen: la prassi, che conosce un sensibile incremento nella Grecia del I secolo d.C., interessa in preponderanza peregrini di condizione modesta (ibid., 21-22). Per il supporto nella ricerca epigrafica, ringrazio F. Camia.

90. Cf. al riguardo Kajanto, 1982, pp. 170 e 128-129 (sui cognomina in -ullus/la). A Roma, sono attestate in epigrafi funerarie di età imperiale sia l'uscita al maschile (EDR093676 del 11-02-2016: A. Carapellucci), sia l'uscita al femminile (5 ricorrenze, tra cui EDR033741 del 09-12-2008: A. Ferraro). 


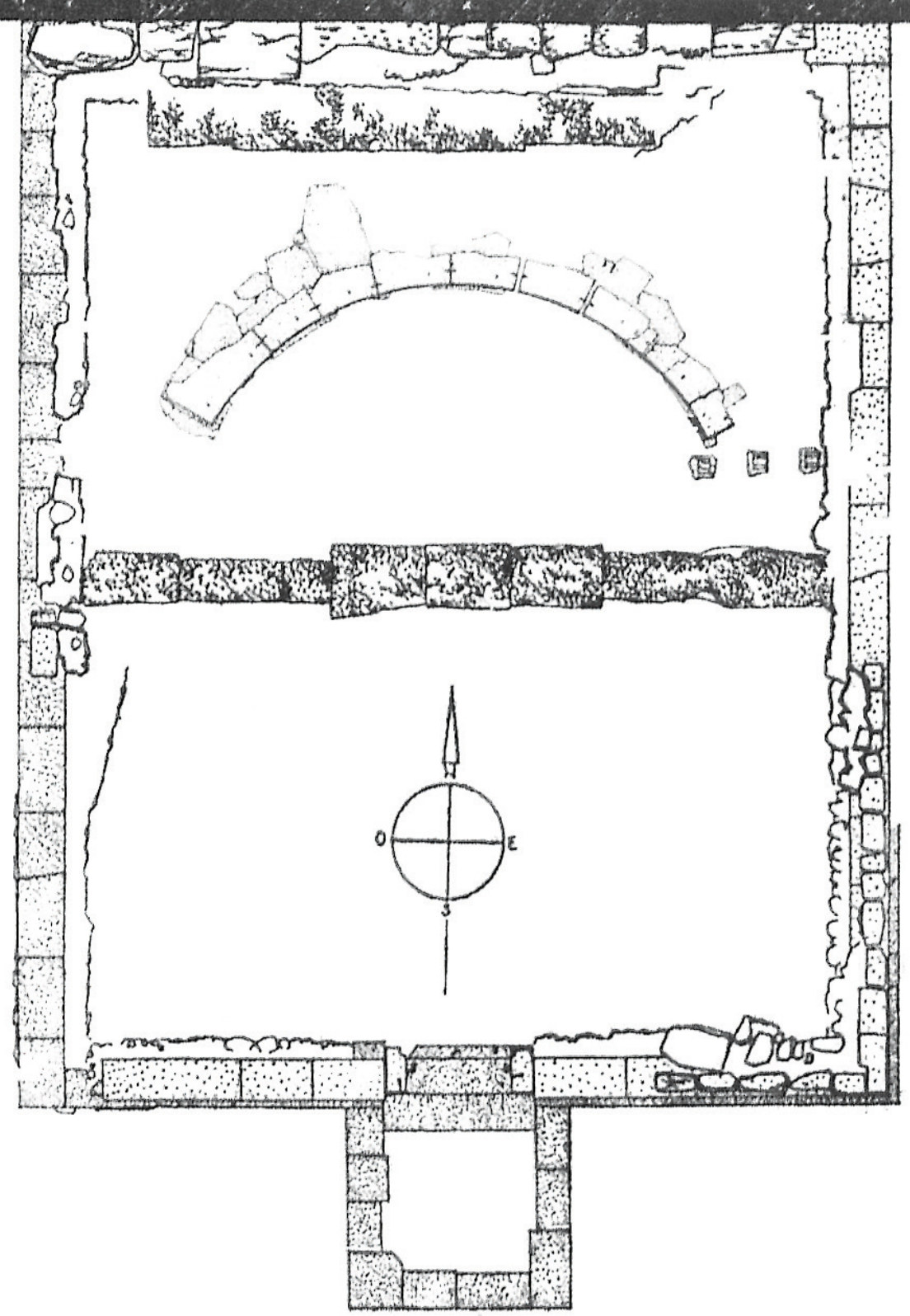

Fig. 5. Epidauro, santuario di Asclepio: edificio $\Omega$ (da Melfi, 2007a) 
inferiore, forse di origini servili, ma di discreta disponibilità economica, considerata la qualità dei doni elargiti al santuario. In ogni modo, stante le informazioni di cui siamo in possesso, la sua origine e la sua precisa condizione giuridica rimangono incerte. L'edificio da lui donato, definito pomposamente nel testo epigrafico un naòs completo di agalma, è un ex voto in seguito a un sogno evidentemente salutifero (દ̌ $\xi$

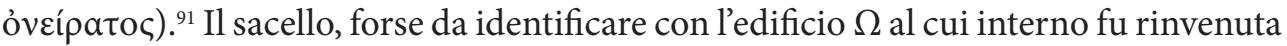
l' iscrizione ${ }^{92}$ (fig. 5), è consacrato a Telesforo, figlio di Asclepio ed egli stesso dio della guarigione, salutato come Soter. Si tratta dunque di una risposta individuale in occasione di una guarigione ottenuta, di sicuro pattuita con la classe sacerdotale: questo edificio segna infatti l' introduzione cultuale di Telesforo nel santuario epidaurio. Più o meno contemporaneamente, Fabullos dedica una statua ad Artemis Ecate, in quanto divinità epekoos. ${ }^{93}$ L'epiteto, ben attestato in età ellenistica, trasmette le qualità di divinità guaritrice e salutifera: grande dunque doveva essere la riconoscenza di Fabullos verso quanti lo avevano aiutato nel percorso individuale di recupero della propria integrità fisica. ${ }^{94}$

Non potrebbero essere più distanti da queste le dediche degli edifici a Mantinea, l'uno consacrato ad Adriano e l'altro dedicato a Antinoo in quanto divinità epicoria.

Aulus Maecius Phaedrus è un cittadino romano non altrimenti attestato, forse di condizione libertina. ${ }^{95}$ La dedica della statua di Adriano insieme al tempio a lui intitolato (tab. 2, cat. 10) è, come già notato da Louis Robert, una summa honoraria: ${ }^{96}$ un pagamento dovuto nel medesimo anno della sua entrata in carica come grammateus. Non si tratta dunque di una forma di devozione, né di una risposta di consenso individuale all' imperatore in carica, ma dell'adesione pubblica a un codice di comportamento ufficiale richiesto dalla sua carica. Il tono dell' iscrizione si adegua alla

91. L'indicazione $\grave{\varepsilon} \xi$ ỏveípatoc è un unicum nel repertorio epigrafico, e deve essere considerata

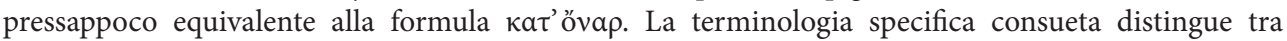

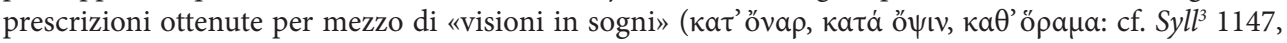

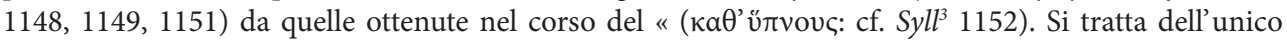
edificio donato a Epidauro con questa motivazione, laddove $\kappa a \tau$ ' óva $\rho$ sono attestate soprattutto dediche di altari, statue o suppellettile (Schörner, 2003, pp. 20-21).

92. L'idea è già di Milena Melfi, espressa in Melfi, 2007b, p. 252.

93. IG IV² 1, 499; RomPel I, p. 191, cat. ARG 123,1; Schörner, 2003, p. 344, cat. 452 con bibl. prec.

94. Sulle divinità epekooi, vd. Weinreich, 1912, p. 912.

95. La formula onomastica del prenome+gentilizio+cognomen greco farebbe pensare a un personaggio affrancato dalla condizione servile. Ma, come segnala Ath. Rizakis (Rizakis, 1996, p. 26) la varietà di soluzioni alternative possibili raccomanda una certa cautela nell' inferire da una formula onomastica una condizione sociale.

96. Sulla formula viđè $\rho \rho \alpha \mu \mu \alpha \tau \dot{a} \alpha \varsigma$, vd. Robert, 1937, pp. 414-415, n. 7; sulla summa honoraria Quaß, 1993, pp. 328-334. 
circostanza, riportando nelle prime righe la titolatura completa di Adriano e sottolineando in chiusura l'esborso integralmente a carico di Maecius.

Ancora differente il caso di C. Iulius Eurykles Herclanus L. Vibullius Pius, membro di una delle più influenti famiglie di Sparta e primo senatore spartano: ${ }^{97}$ al suo

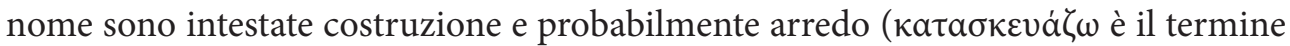
impiegato nel testo epigrafico) a Mantinea di una stoà con esedre dedicata alla città e insieme ad Antinoo in quanto divinità epicoria ${ }^{98}$ (tab. 2, cat. 11). In calce, si legge che la dedica fu espletata $\delta ı \grave{\alpha} \tau \tilde{\omega} \nu \kappa \lambda \eta \rho o v o ́ \mu \omega v$ : l'indicazione, un unicum nel repertorio epigrafico, indica che la dedica e forse la costruzione dell'edificio furono ottemperate alla sua morte dagli eredi, evidentemente desiderosi di confermare lo status e il peso politico della famiglia. Si tratta di una novità straordinaria, essendo l'unico edificio nel Peloponneso consacrato espressamente alla memoria di Antinoo in questi decenni. La scelta, solo apparentemente singolare, si inserisce nell'alveo di una particolare attenzione che lo stesso Adriano aveva riservato alla città di Mantinea, ritenuta la madrepatria di Bithynion, e per ciò stesso strettamente correlata alla persona di An-

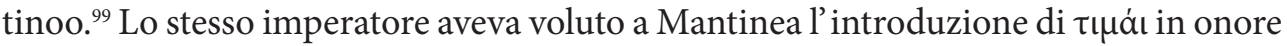
del giovinetto, che avevano preso la forma di un culto misterico a cadenza annuale e agoni isolimpici ginnici a cadenza penteterica. ${ }^{100}$

A Tegea, l'unico edificio eretto da un privato è un oikos dedicato agli inizi del II secolo d.C. a tutti gli dei per volontà di P. Memmios Agathokles, a conclusione del suo ufficio di agoranomos (tab. 2, cat. 13). All' interno del tempietto erano custoditi, sotto lo sguardo vigile degli dei, i pesi in bronzo utilizzati nel corso della carica, accuratamente elencati insieme al loro peso (espresso secondo il sistema romano in litra e unciae) nell' iscrizione dedicatoria. La formulazione del testo porterebbe a ritenere che si tratti di un vero e proprio edificio consacrato, una sorta di sacello o tempietto: si legge chiaramente infatti che Memmios dedica l'oũ $o v \pi[a ́ v \tau \omega \nu \theta \varepsilon] \tilde{\omega} \nu$, con indicazione al genitivo dell' intestazione della proprietà dell'edificio. Nel mondo greco e

97. Halfmann, 1979, pp. 125-126, n. 29; RomPel I, 134-135; RomPel II, 292-293; Rizakis e Zoumbaki, 2017, p. 168 , n. 46.

98. L'iscrizione con la dedica è incisa sull'architrave forse pertinente a una edicola al cui interno è verosimile immaginare una statua dello stesso Iulius Herclanus, morto nel 136/137 d.C. (in tal senso, già Meyer, 191, p. 200).

99. Paus., VIII 9, 7. Un recentissimo intervento di James Roy riferisce a una volontà dello stesso Adriano l'ideazione del legame di filiazione Mantinea-Bithynion (Roy, 2016, pp. 120-123, 127, con bibl. prec.).

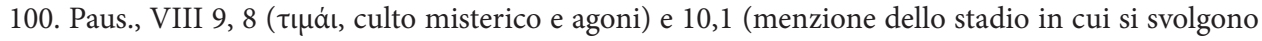
gli agoni per Antinoo); IG V 2313 (RomPel I, Arc 3): attestazione di un agonotheta degli Antinoeia, tale

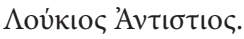


latino era prassi comune la custodia dei pesi pubblici all' interno di templi dedicati agli dei che, simbolicamente, ne avrebbero sorvegliato la custodia. ${ }^{101}$

Bisognerà attendere la fine del secolo, o forse già gli inizi del successivo, per imbattersi nelle ultime due dediche per interessamento di personaggi locali, e curiosamente si tratta di due triclini. In Messenia, nel santuario di Apollo Korythos, ${ }^{102}$ il sacerdote uscente Flavios Alkimos (tab. 2, cat. 9) offre a proprie spese un triclinio che, così recita l'iscrizione, poteva essere utilizzato anche dagli i $\varepsilon \rho \omega \dot{\omega} \mu \varepsilon v o l$, gli altri sacerdoti o forse i consacrati. ${ }^{103}$ Doveva essere dunque un ambiente riservato al pasto comune in ottemperanza al rituale, di cui ignoriamo però la dinamica specifica. $\grave{E}$ ancora un sacerdote, ma in carica, a curare l'erezione di una stoà e di un triclinio nel santuario inferiore di Asclepio al Gortynios, in piena Arcadia (tab. 2, cat. 5). Ne conosciamo il nome, Marcos Tourpilios Philotas, greco e cittadino romano. Curiosamente, egli non è però un evergeta nel senso tradizionale del termine: i due edifici, così recita testualmente l'iscrizione, sono infatti finanziati grazie alle entrate nella cassa sacra del dio. In qualche modo, è Asclepio che finanzia sé stesso. Tourpilios si limita, verosimilmente, a seguire i lavori e a far incidere il proprio nome, in lettere apicate, al nominativo, alla stregua di un vero benefattore. ${ }^{104}$

E veniamo al ruolo dei senatori. Solo pochi sono i senatori espressione delle più ricche famiglie del Peloponneso nel II secolo d.C. A Sparta, oltre al già citato C. Iulius Eurycles Herculanus, il solo Ti. Claudius Brasidas; ${ }^{105}$ a Messene Ti. Claudius Frontinus ${ }^{106}$ e i suoi figli (Ti. Claudius Frontinus Niceratus ${ }^{107}$ e Ti. Claudius Saethida Caelianus: ${ }^{108}$ solo Ti. Claudius Frontinus riuscì a divenire console, nel 149/151

101. A mio avviso, non vi è pertanto ragione di credere, come propone G. Schörner, che l' oikos non sia un edificio di culto in sé ma una sorta di scrigno consacrato e deputato alla custodia degli oggetti ormai dismessi (Schörner, 2003, p. 126).

102. Sul santuario, individuato in località Petalidion a sud di Korone, vd. Lo Monaco, 2009, in part. pp. 78-80, con fig. 33, e pp. 726-728, cat. Mess. Koryth. 1. Gli scavi, parziali e limitati a una sola campagna nell'anno 1915, non hanno restituito alcun edificio interpretabile come triclinio.

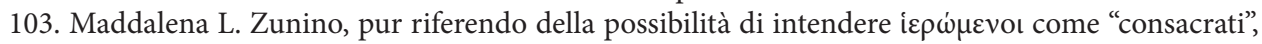
preferisce però tradurre il participio con "sacerdoti" (così, nella sua traduzione del testo: Zunino, 1997, p. 151, T 42). Nella sua disamina a p. 177, ipotizza per il triclinio un uso nel corso di celebrazioni iniziatiche e misteriche.

104. P. Amandry in BCH 1942/1943, p. 336, fig. 13.

105. Halfmann, 1979, p. 188, n. 111 (età di Marco Aurelio); RomPel II, Lac 274; Rizakis e Zoumbaki, 2017, p. 169 (età di Adriano o al più tardi di Antonino Pio).

106. Halfmann, 1979, p. 174, n. 93 (il padre ai tempi di Antonino Pio); RomPel II 142;

107. Halfmann, 1979, p. 196, n. 126.

108. Halfmann, 1979, p. 196, n. 127.

ARYS, 16, 2018 [139-176] ISSN 1575-166X 


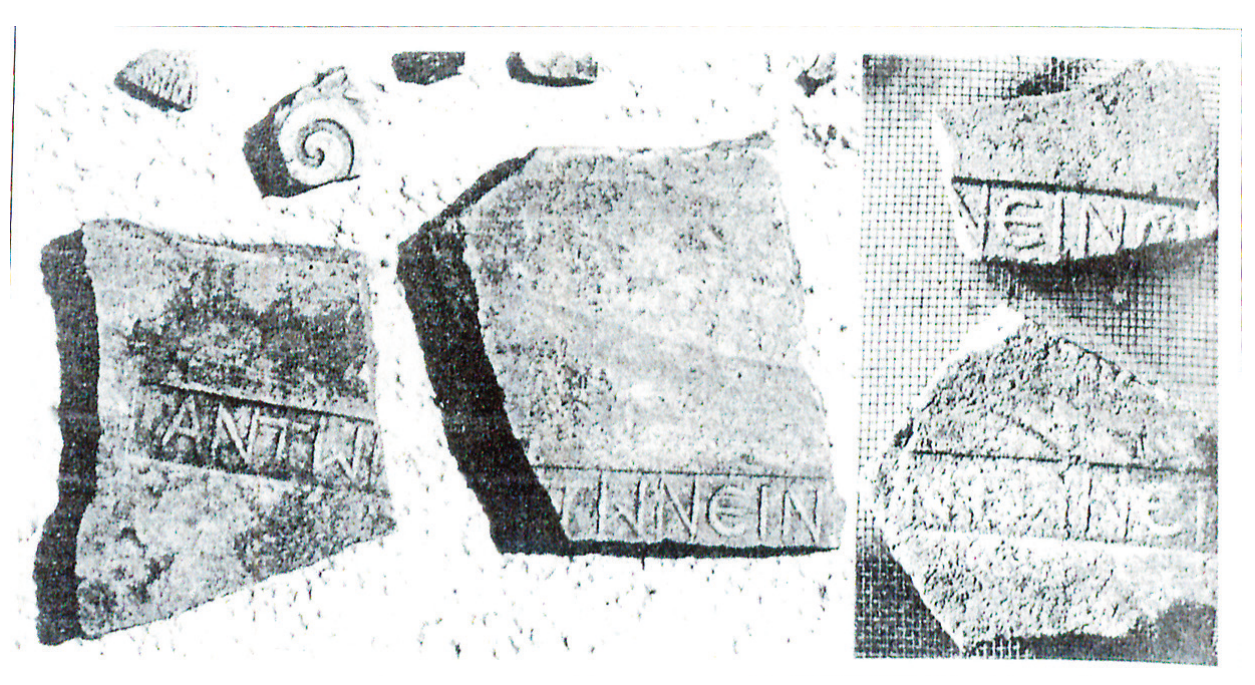

Fig. 6. Epidauro, santuario di Asclepio: tegole con il bollo ANTSNEIN (da Vitti, 2016)

o tra il 155 e il 160). ${ }^{109}$ Molto ipotetica la provenienza da Corinto di Veturius Paccianus. ${ }^{110}$ L'unico a essere coinvolto in un atto evergetico che abbia una qualche relazione con il sacro è, come detto, C. Iulius Eurycles Herculanus a Mantinea. Né a Messene né a Corinto i senatori locali sembrano coinvolti in operazioni di finanziamento di edifici sacri o all' interno di aree sacre. Il dato è tanto più stupefacente qualora si consideri come alcuni di essi abbiano contestualmente ricoperto l'ufficio di archiereus a vita dei Sebastoi (C. Iulius Eurycles Herculanus, Ti. Claudius Frontinus) ${ }^{111}$ o di sodalis Hadrianalis (Ti. Claudius Frontinus e i suoi figli, Niceratus e Saethida Caelianus), indici di una buona dimestichezza con le prassi inerenti al culto imperiale e più in generale con la gestione degli spazi sacri e delle relative cerimonie. Anche il computo dei senatori di altra provenienza attivi in qualità di evergeti in ambito sacro nel Peloponneso offre qualche sorpresa, lasciandoci re-

109. Da ultimo, cf. l'analisi di Rizakis, 2009.

110. Halfmann, 1979, p. 192, n. 118 (ai tempi di Marco Aurelio). Sul personaggio, da ultimo Zoumbaki, 2008, p. 47, n. 87 , con bibl. prec. e discussione della provenienza solo indiziaria del personaggio da Corinto.

111. IG V 11455. 
gistrare i soli casi del facoltoso senatore originario di Nysa, Sextus Iulius Maior Antoninus Pythodorus (tab. 2, cat. 1-2), cui si devono vari edifici templari e infrastrutture nei due santuari di Epidauro ${ }^{112} \mathrm{e}$ dell'ateniese Erode Attico (tab. 2, cat. 8, 12), ${ }^{113}$ finanziatore a Olimpia del sontuoso ninfeo nei pressi dell'Heraion e dell'acquedotto necessario per alimentarlo e a Isthmia delle statue colossali di Poseidon e Anfitrite. L'intensa attività di entrambi ci permette una riflessione ulteriore. Nei loro cantieri si utilizzano infatti laterizi prodotti per l'occasione. A Epidauro ne è testimonianza una nutrita serie di bolli su tegola, con la scritta ANTSNEIN (fig. 6), rinvenuta in due edifici all' interno dell'Asklepieion (edifici $\Phi$ e K), nel complesso presso la cisterna dell'Asklepieion e nella cd. skanà sul Kynortion; ;14 a Olimpia, alcuni laterizi bollati recano l'indicazione al genitivo, entro cartiglio rettangolare, del nome di Erode Attico. ${ }^{115}$ È verosimile immaginare che le figlinae fossero a non eccessiva distanza dai cantieri: avremmo dunque a che fare con una organizzazione complessiva a largo raggio, di portata decisamente inusuale, che non si limita al finanziamento del singolo edificio, ma che comporta l'impianto di attività produttive in loco. Il dato è ancora più interessante qualora si noti come entrambi i senatori in questione siano di provenienza alloctona, originari di Nysa uno, di Atene l'altro. Tali attività lasciano dunque intravedere un'ottima rete di relazioni locali.

A prescindere da questi casi, eclatanti ma sporadici e talora persino capaci di attirare lo scontento delle classi meno abbienti, ${ }^{116}$ più in generale direi che il peso dei senatori sulla vita dei santuari piccoli e grandi amministrati dalle cittadine locali non dovette essere soverchiante. A fronte di tale apparente disinteresse, bisogna rilevare come la vita delle poleis fosse ancora nelle mani non tanto dei senatori quanto degli esponenti delle vecchie famiglie aristocratiche, capaci di mantenere ricchezza

112. Il programma evergetico del senatore Antoninus Pythodorus è stato oggetto in anni recenti di approfondite disamine. Tra gli interventi più attenti, con bibl. prec., segnalo Galli, 2001, pp. 52-56 e 2004, pp. 328-344; Melfi, 2007a, pp. 121-123; 2007b, pp. 248-252; 2010, pp. 334-338.

113. Sull'attività di Erode Attico come ervegeta si vedano Galli, 2002 e da ultimo Rizakis, 2015, pp. 146-147, n. 15 (con bibl. prec.).

114. Secondo Panagiotis Kavvadias, nel 1900 al Museo di Epidauro erano custoditi 12 frammenti di tegola con il bollo ANT $2 N E I N$ (Kavvadias, 1900, p. 157). Al riguardo cf. Galli, 2004, p. 330; Melfi, 2007b, p. 248; Vitti, 2016a, p. 186 e fig. 3.117.

115. Si tratta di un laterizio con bollo entro cartiglio rettangolare: ArchZeit 1876, p. 59 (W. Dittenberger); Ginouvès, 1972, p. 217; Galli, 2004, p. 330; Vitti, 2016a, p. 78. Per l'acquedotto, cf. Luc., De mort. Peregr. 19, 20; Philostr., VS II 1, 5.

116. Si veda l'ostilità di Peregrino riguardo alla costruzione del Ninfeo di Erode Attico a Olimpia, reo, a suo dire, di svuotare l'aura sacrale del luogo (Settis, 1968, p. 29). Sulle classi meno agiate e le loro aspettative legate a bisogni più immediati cf. Barresi, 2007, p. 140 con il commento delle lamentele degli efesini contro Vedio Antonino, impegnato nella costruzione di varie opere pubbliche in città. 
e influenza cittadina per oltre un secolo, ${ }^{117}$ forti anche di relazioni reciproche a livello sovra-urbano e di una certa facilità nel dialogo politico con la classe governativa romana. ${ }^{118}$ Alcuni dei loro membri ancora nel II secolo d.C. sono membri dell' ordo decurionum, organo cui erano demandati, tra gli altri compiti, anche costruzione e restauro dei monumenti pubblici. ${ }^{119} \mathrm{E}$ dunque verosimile che una buona percentuale dei cantieri di restauro più o meno estensivo di cui non conosciamo i finanziatori (vd. tab. 3) fossero appaltati e remunerati a livello cittadino. Il supporto finanziario di senatori e di membri delle élites cittadine sembra di preferenza rivolto a opere di pubblica utilità o di abbellimento dei centri urbani, con la costruzione di stadi, ginnasi, ponti, acquedotti e altri edifici di carattere pubblico. ${ }^{120}$ I santuari, almeno nel Peloponneso, restano nell'ombra.

\section{Conclusioni}

L'esame in diacronia delle attività edilizie nei santuari del Peloponneso nel II secolo d.C. offre qualche sorpresa. Stupisce in prima battuta una certa esiguità di interventi, se paragonati alla natura e alla quantità degli atti evergetici che interessano pressappoco contemporaneamente i centri abitati. Le erezioni di nuovi edifici templari sono ridotte al minimo, rarissimi sono gli interventi edilizi di carattere monumentale, e la maggior parte delle attività è inerente al restauro e alla manutenzione ordinaria degli edifici.

Qualche sopresa anche sul fronte dei committenti.

Tra gli imperatori in carica, l'unico a finanziare in prima persona l'avvio di cantieri (di restauro, nella logica però del rifacimento integrale), è Adriano (tab. 1). Gli altri imperatori sono del tutto assenti. Eppure, nonostante una certa assiduità di Adriano con l'Ellade (mai eguagliata), la sua presenza non diviene occasione immediata per un restyling e ammodernamento dei santuari. Gli onori a lui conferiti nel corso delle visite si limitano all'erezione di statue in suo onore, corredate da epiteti enfatici e del tutto convenzionali (Epidauro), ${ }^{121}$ ma non si traducono in interventi di maquillage tesi a migliorare la qualità architettonica degli spazi sacri.

La bassa partecipazione evergetica di cittadini abbienti nei confronti dei sacra nella prima metà del II secolo d.C. non può che sorprendere, se paragonato a una più attiva e quasi capillare presenza di cittadini che, nel Peloponneso già del I secolo a.C.,

117. Sulle élites del Peloponneso, cf. Zoumbaki, 2010; Rizakis e Zoumbaki, 2017, p. 169.

118. Rizakis, 2015, pp. 147-149.

119. Sui compiti demandati all' ordo decurionum in Campania, vd. Camodeca, 2003.

120. Rizakis, 2007, pp. 317-318.

121. Lo Monaco, 2014, p. 29. 
si erano fatti carico del restauro di edifici templari o dell'assunzione dei costosi sacerdozi. ${ }^{122}$ Stupisce poi non poco l'assenza delle donne, ai cui nomi non è associato nessuno dei pur esigui atti avergetici del periodo, con la sola eccezione di Annia Regilla, intestataria nominale dello splendido ninfeo finanziato a Olimpia dal marito (tab. 2, cat. 12). È un contrasto molto forte se paragonato alle gesta di facoltose benefattrici registrate tra il I secolo a.C. e il I secolo d.C. in Arcadia, che erano si fatte carico "senza esitazione", ${ }^{123}$ del restauro di edifici templari e dello svolgimento delle cerimonie, ${ }^{124}$ dell'organizzazione di banchetti, ${ }^{125}$ del dono di interi appezzamenti di terreno coltivato ${ }^{126} \mathrm{O}$, più semplicemente, della dedica di suppellettile cultuale di un certo pregio. ${ }^{127}$ Insieme ai loro nomi, spariscono quasi del tutto anche le dediche delle loro statue e dei loro ritratti, dipinti e talora persino dorati, all'interno dei naoi. ${ }^{128}$ Bisognerà attendere ancora qualche decennio per avere, a partire dagli anni Sessanta del II secolo, ingenti finanziamenti da parte di ricchi evergeti privati (Antoninus Pythodorus, Iuventianus, Erode Attico), senatori o membri delle più eminenti famiglie locali, che permetteranno finalmente atti di grande respiro, frutto di precise scelte individuali ma certamente molto gradite al clero locale per la capacità di interagire con spazi sacri dalla frequentazione centenaria, ora profondamente ammodernati nell'assetto monumentale complessivo e talora persino nei percorsi processionali.

122. Lafond, 2016, in part. pp. 19-20.

123. Commenta ottimamente la retorica della formulazione Lafond, 2016, in part. p. 24.

124. Nikippa figlia di Pasias a Mantinea nel I sec. a.C. si incarica dei lavori necessari al tempio di Kore e delle relative liturgie e sacrifici ( $I G$ V,2 265; Lo Monaco, 2009, 371-373, cat. Arc. Mant. 25); Phaena di Damatrios finanzia negli anni Quaranta del I secolo a.C. il restauro del megaron di Kore e le feste (IG V,2 266: Lo Monaco, 2009, 373-375, cat. Arc. Mant. 26).

125. Kleinika, all'Eleusion poco fuori Sparta (IG V,1 229; Lo Monaco, 2009, p. 603, cat. Lac. Ele 7).

126. Giulia Eudia a Mantinea nel corso del I sec. d.C. dona al santuario 14 iugera di vitigni di Zeus Epidotes e 6 iugera ad Asclepio. Sue immagini sono erette in ringraziamento all' interno dei templi (IG V,2 270: Lo Monaco, 2009, p. 358, cat. Arc. Mant. 4; IG V,2 269: Lo Monaco, 2009, pp. 365-367, cat. Arc. Mant. 14).

127. Lykosoura: trapeza marmorea dedicata alla Despoina dalla sua sacerdotessa uscente, Ainesos figlia di Erasippos (IG V,2 521; Lo Monaco, 2009, pp. 341-342, cat. Arc. Lyk 5).

128. Santuario della Despoina a Lykosoura: Timasistrata figlia di Onasikrates (Lo Monaco, 2009, pp. 346-347, cat. Arc. Lyk 10); Giulia Pantimia (Lo Monaco, 2009, p. 350 cat. Arc. Lyk 16); la figlia di Aristokrates (Lo Monaco, 2009, p. 351 cat. Arc. Lyk 18). Pronao dell'Heraion di Olimpia: Klaudia Alkinoa, Numisia Teisis e Antonia Kleodike ( $I v O$ 435, 438, 429; Lo Monaco, 2009, p. 565, cat. El. Ol. 85; pp. 571-572, , cat. El. Ol. 94; p. 573, cat. El. Ol. 96). Fa eccezione la sola statua onoraria in favore di Volusena Iusta, esposta intorno al 150 d.C. all' interno del pronao del tempio della Despoina a Lykosoura: la ragione della sua dedica, ascritta alla sua areté, è purtroppo molto generica (IG V,2 544; RomPel I, Arc 172).

ARYS, 16, 2018 [139-176] ISSN 1575-166X 
Un'ultima osservazione sui luoghi cui sono destinate le risorse investite: se l'attività dei ricchi evergeti di età antoniniana interessa come è ovvio in esclusiva i santuari panellenici (Isthmia, Olimpia) o almeno a intensa frequentazione (Epidauro), i cittadini privati sembrano privilegiare i sacra locali della propria comunità, ove dedicano e consacrano agli dei piccoli sacelli e oikoi (tab. 2, cat. 3, 10, 13). Credo non sia interamente dovuto al caso se sono per noi anonimi gli interventi di restauro e manutenzione straordinaria registrati negli edifici templari di numerose poleis del Peloponneso (tab. 3): necessari, ma non abbastanza altisonanti per trascinare con sé memoria e fama futura dei finanziatori, essi poterono essere forse intrapresi con il ricorso alle casse cittadine, evidentemente ancora capaci di singoli capitolati di spesa.

TABELLA 1. Templi o edifici dedicati o restaurati in santuari da imperatori

\begin{tabular}{|l|l|l|l|l|l|}
\hline & LUOGO & EDIFICIO & IMPERATORE & FONTE & BIBL. \\
\hline 1. & Argo & $\begin{array}{l}\text { tempio di } \\
\text { Hera (Akraia } \\
\text { o Antheia?): } \\
\text { restauro }\end{array}$ & $\begin{array}{l}\text { Adriano, prima } \\
\text { di Aprile del } \\
128\end{array}$ & $\begin{array}{l}\text { SEG XI } \\
340\end{array}$ & Piérart, 1995 \\
\hline 2. & Mantinea & $\begin{array}{l}\text { Santuario } \\
\text { di Poseidon } \\
\text { Hippios: } \\
\text { ricostruzione }\end{array}$ & Adriano & $\begin{array}{l}\text { Paus., } \\
\text { VIII } \\
10,2\end{array}$ & $\begin{array}{l}\text { Lo Monaco, 2014, pp. 29- } \\
30\end{array}$ \\
\hline
\end{tabular}




\begin{tabular}{|c|c|c|c|c|c|}
\hline & LOCALITÀ & $\begin{array}{l}\text { NOME } \\
\text { DELL'EVER- } \\
\text { GETA }\end{array}$ & $\begin{array}{l}\text { CITTÀ } \\
\text { DI PRO- } \\
\text { VENIEN- } \\
\text { ZA }\end{array}$ & DATA & BIBL. \\
\hline 1. & $\begin{array}{l}\text { Epidauro, } \\
\text { Asklepieion. } \\
\text { Bagno di Asclepio, } \\
\text { santuario degli dei } \\
\text { Epidotai, tempio } \\
\text { per Hygea, Asclepio } \\
\text { e Apollo Egizi. } \\
\text { Ricostruzione del } \\
\text { portico di Kotys. } \\
\text { Katagogion. } \\
\text { Santuario di Apollo } \\
\text { Maleatas: opere e } \\
\text { serbatoio idrico }\end{array}$ & $\begin{array}{l}\text { Senatore } \\
\text { romano } \\
\text { Antoninus } \\
\text { (=Sextus } \\
\text { Iulius Maior } \\
\text { Antoninus } \\
\text { Pythodorus: } \\
\text { tab. 2, cat. 2) }\end{array}$ & Nysa? & & $\begin{array}{l}\text { Paus., II 27, 6; Lafond, } \\
\text { 2006, p. } 245\end{array}$ \\
\hline 2. & $\begin{array}{l}\text { Epidauro, } \\
\text { Asklepieion: } \\
\text { ricostruzione delle } \\
\text { terme; restauro di } \\
\text { edifici. } \\
\text { Sant. di Apollo } \\
\text { Maleatas: restauro } \\
\text { di edifici. }\end{array}$ & $\begin{array}{l}\text { Sextus } \\
\text { Iulius Maior } \\
\text { Antoninus } \\
\text { Pythodorus }\end{array}$ & Nysa & & $\begin{array}{l}\text { IG IV } \text { IV }^{2} 454 \text {; } \\
\text { Halfmann, 1979, pp. } \\
\text { 171-172, n. 89; RomPel } \\
\text { I, pp. 199-200, cat. } \\
\text { ARG 149; Galli, 2002; } \\
\text { Melfi 2007a, pp. 99- } \\
\text { 123; Di Napoli, 2013, } \\
\text { p. 51; Yegül, 2015, pp. } \\
\text { 256-258 e fig. 12.11; } \\
\text { Vitti, 2016a, pp. 186- } \\
\text { 192. }\end{array}$ \\
\hline 3. & $\begin{array}{l}\text { Epidauro, } \\
\text { Asklepieion: dedica } \\
\text { a Telesforo Soter } \\
\text { di un naos e di un } \\
\text { agalma }\end{array}$ & Fabullos & & $117-138$ & $\begin{array}{l}\text { IG IV } \text { IV 561; Peek, } \\
\text { 1969, n. 235; RomPel } \\
\text { I, 191, cat. ARG 123; } \\
\text { Schörner, 2003, n. 537; } \\
\text { Melfi, 2007a, tab. 7, n. } \\
\text { 456. }\end{array}$ \\
\hline 4. & $\begin{array}{l}\text { Epidauro, } \\
\text { Asklepieion, } \\
\text { biblioteca e libri }\end{array}$ & Gaios Roufos & $\begin{array}{l}\text { Napoli } \\
\text { (?) }\end{array}$ & II d.C. & $\begin{array}{l}\text { IG IV }{ }^{2} \text { 1, } 456 \text { + IG IV } \\
\text { 1011; Schörner, 2003, } \\
\text { n. 538; Perilli, 2006, p. } \\
\text { 491; Melfi, 2007a, tab. } \\
\text { 7, n. } 496\end{array}$ \\
\hline
\end{tabular}




\begin{tabular}{|c|c|c|c|c|c|}
\hline 5. & $\begin{array}{l}\text { Gortina (Arcadia), } \\
\text { Asklepieion, stoà e } \\
\text { triclinio }\end{array}$ & $\begin{array}{l}\text { (Marcos } \\
\text { Tourpilios } \\
\text { Philotas) }\end{array}$ & & $\begin{array}{l}\text { II/III } \\
\text { secolo } \\
\text { d.C. }\end{array}$ & $\begin{array}{l}\text { SEG XI 1165; RomPel } \\
\text { I, 147-148, cat. ARC } \\
\text { 162; Melfi, 2007a, } 219 \text { e } \\
\text { 2007b, } 241\end{array}$ \\
\hline 6. & $\begin{array}{l}\text { Isthmia, Sant. di } \\
\text { Poseidon: } \\
\text { alloggi per atleti; } \\
\text { Palaimonion, altare } \\
\text { degli dei patrii (con } \\
\text { recinto e pronao, } \\
\text { naos di Helios } \\
\text { (con agalma e } \\
\text { peribolo), periboli, } \\
\text { naoi e agalmata di } \\
\text { Demetra e Kore, } \\
\text { Dionysos e Artemis, } \\
\text { templi di (Demeter) } \\
\text { Eueteria, Kore e } \\
\text { del Ploutoneion, } \\
\text { anabaseis e } \\
\text { analemmata (dello } \\
\text { stadio?) in rovina, } \\
\text { portico vicino allo } \\
\text { stadio, con le stanze } \\
\text { e gli arredi }\end{array}$ & $\begin{array}{l}\text { P. Licinius } \\
\text { Priscus } \\
\text { Iuventianus }\end{array}$ & Corinto & $161-169$ & $\begin{array}{l}\text { IG IV 203; Galli, 2002, } \\
\text { pp. } 246-247 \text {; Lafond, } \\
\text { 2006, p. } 244 \text {; Torelli, } \\
2010 \text {, p. } 569\end{array}$ \\
\hline 7. & $\begin{array}{l}\text { Isthmia, Sant. di } \\
\text { Poseidon: } \\
\text { stoà Reguliana }\end{array}$ & $\begin{array}{l}\text { P. Licinius } \\
\text { Priscus } \\
\text { Iuventianus }\end{array}$ & Corinto & $170-175$ & $\begin{array}{l}\text { IG IV } 203 \text {; Torelli } 2010 \text {, } \\
\text { pp. } 572 \text { e } 576 .\end{array}$ \\
\hline 8. & $\begin{array}{l}\text { Isthmia, sant di } \\
\text { Poseidon: statue } \\
\text { colossali di } \\
\text { Poseidon Isthmios e } \\
\text { Anfitrite }\end{array}$ & Erode Attico & & & $\begin{array}{l}\text { Paus., II 1, 7-9; Quaß } \\
\text { 1993, p. 222; Galli } \\
\text { 2002, pp. 214-218; } \\
\text { Torelli 2010, pp. 557, } \\
\text { 581. }\end{array}$ \\
\hline 9. & $\begin{array}{l}\text { Korone, sant. di } \\
\text { Apollo Korythos, } \\
\text { triclinio }\end{array}$ & $\begin{array}{l}\text { Flavius } \\
\text { Alkimos }\end{array}$ & & $153-154$ & $\begin{array}{l}\text { RomPel II, 533, cat. } \\
\text { MES 188; Schörner, } \\
\text { 2003, n. } 768 .\end{array}$ \\
\hline 10. & $\begin{array}{l}\text { Mantinea, statua e } \\
\text { tempio per Adriano }\end{array}$ & $\begin{array}{l}\text { A(ulus) } \\
\text { Maecius } \\
\text { Phaedrus }\end{array}$ & Mantinea & $125-128$ & $\begin{array}{l}\text { IG V,2 302; Jost, } 1985 \text {, } \\
\text { p. } 130 ; \text { RomPel I, } 137 \\
\text { cat. ARC } 117 .\end{array}$ \\
\hline
\end{tabular}




\begin{tabular}{|c|c|c|c|c|c|}
\hline 11. & $\begin{array}{l}\text { Mantinea, stoà } \\
\text { dedicata ad } \\
\text { Antinoo come } \\
\text { divinità epicoria }\end{array}$ & $\begin{array}{l}\text { C. Iulius } \\
\text { Eurykles } \\
\text { Herclanus L. } \\
\text { Vibullius Pius }\end{array}$ & Sparta & $136-137$ & $\begin{array}{l}\text { IG V,2 281; Halfmann, } \\
\text { 1979, pp. 125-126, n. } \\
\text { 29; Jost, 1985, pp. 128- } \\
\text { 129; RomPel I, 134-135, } \\
\text { cat. ARC }{ }^{*} 105 ; \text { RomPel } \\
\text { II, 286-294 cat. LAC } \\
\star 462 .\end{array}$ \\
\hline 12. & Olimpia, ninfeo & $\begin{array}{l}\text { (Erode } \\
\text { Attico) Annia } \\
\text { Regilla }\end{array}$ & & 175 & $\begin{array}{l}\text { IvO 610; Bol, 1984; } \\
\text { RomPel I, 418-420, } \\
\text { cat. EL 17; Galli, 2002, } \\
\text { pp. 222-227; Schörner, } \\
\text { 2003, pp. 120-121. }\end{array}$ \\
\hline 13. & $\begin{array}{l}\text { Tegea, oikos e pesi } \\
\text { in bronzo }\end{array}$ & $\begin{array}{l}\text { Poplios } \\
\text { Memmios } \\
\text { Agathokles }\end{array}$ & & $\begin{array}{l}\text { Età di } \\
\text { Traiano } \\
\text { o } \\
\text { Adriano }\end{array}$ & $\begin{array}{l}\text { IG V,2 125; Jost, } 1985 \text {, } \\
\text { 522; RomPel I, } 138 \text { cat. } \\
\text { ARC 122; Schörner, } \\
\text { 2003, cat. } 812 .\end{array}$ \\
\hline
\end{tabular}

\begin{tabular}{|c|c|c|c|c|}
\hline & LUOGO & EDIFICIO & DATA & BIBL. \\
\hline 1. & Egira & $\begin{array}{l}\text { Tempio di Artemis. Forse } \\
\text { da identificare con un } \\
\text { piccolo prostilo ionico } \\
\text { tetrastilo (ed. E): restauri }\end{array}$ & $\begin{array}{l}\text { II secolo d.C. (età } \\
\text { antoniniana?) }\end{array}$ & $\begin{array}{l}\text { Paus., VII 26, 5; Gogos, } \\
\text { 1986-1987. }\end{array}$ \\
\hline 2. & Argo & $\begin{array}{l}\text { Santuario di Apollo Pizio } \\
\text { Teatro }\end{array}$ & & Di Napoli, 2013, p. 51 \\
\hline 3. & Argo & $\begin{array}{l}\text { Santuario di Asclepio } \\
\text { Terme }\end{array}$ & II secolo d.C. & $\begin{array}{l}\text { Vitti, 2010; Ottati, } \\
\text { 2016, pp. 242-245. }\end{array}$ \\
\hline 4. & Argo & $\begin{array}{l}\text { Heraion, } \\
\text { Terme romane }\end{array}$ & & $\begin{array}{l}\text { Galli, } 2004 \text {, p. } 347 \text { e } \\
\text { fig. } 23 .\end{array}$ \\
\hline 5. & Corinto & Tempio J: nuova erezione & $\begin{array}{l}\text { 183-185 (Grosso); } \\
191 \text { (Brown) }\end{array}$ & $\begin{array}{l}\text { Grosso, 1964, p. 534; } \\
\text { Brown, } 2018 .\end{array}$ \\
\hline
\end{tabular}

129. Nella selezione del materiale, non si è tenuto conto dei restauri indicati nell'edito come genericamente pertinenti fasi di "età romana" o di "età imperiale". 


\begin{tabular}{|c|c|c|c|c|}
\hline 6. & Corinto & $\begin{array}{l}\text { Tempio H: nuova erezio- } \\
\text { ne }\end{array}$ & $\begin{array}{l}183-185 / 191 \\
\text { (Grosso); } \\
191 \text { (Brown) }\end{array}$ & $\begin{array}{l}\text { Grosso, 1964, p. 534; } \\
\text { Brown, } 2018 .\end{array}$ \\
\hline 7. & Corinto & Asklepieion: restauro & 138-212 d.C. & Melfi, 2007a, p. 241. \\
\hline 9. & Epidauro & $\begin{array}{l}\text { Asklepieion: Odeion: } \\
\text { nuova erezione }\end{array}$ & $\begin{array}{l}\text { Inizi del II secolo } \\
\text { d.C. }\end{array}$ & $\begin{array}{l}\text { Bressan, 2009, pp. 99- } \\
\text { 101; Di Napoli, 2013, } \\
\text { pp. 60-61; Vitti, 2016a, } \\
\text { pp. 171-174. }\end{array}$ \\
\hline 10. & Ermione & $\begin{array}{l}\text { Sant. di Demetra: nuova } \\
\text { erezione }\end{array}$ & & IG IV 743. \\
\hline 11. & Isthmia & $\begin{array}{l}\text { Tempio e recinto per Pa- } \\
\text { laimon a est del tempio: } \\
\text { nuova erezione }\end{array}$ & Età adrianea & Gebhard, 1991. \\
\hline 12. & Isthmia & $\begin{array}{l}\text { Sant. di Poseidon } \\
\text { Portico N }\end{array}$ & Età antoniniana & Torelli, 2010. \\
\hline 13. & Isthmia & $\begin{array}{l}\text { Sant. di Poseidon } \\
\text { Stadio }\end{array}$ & & $\begin{array}{l}\text { Gebhard, 1991, Di } \\
\text { Napoli, 2013, p. } 50 .\end{array}$ \\
\hline 14. & Isthmia & $\begin{array}{l}\text { Sant. di Poseidon } \\
\text { Teatro: lavori per amplia- } \\
\text { re la cavea }\end{array}$ & Metà del II secolo & $\begin{array}{l}\text { Paus., II 1, 7; Bressan, } \\
\text { 2009, pp. 148-157; } \\
\text { Di Napoli, 2013, pp. } \\
\text { 49-51. }\end{array}$ \\
\hline \multirow[t]{2}{*}{15.} & Isthmia & $\begin{array}{l}\text { Sant. di Poseidon, terme: } \\
\text { nuova erezione }\end{array}$ & $\begin{array}{l}\text { Metà del II secolo } \\
\text { d.C. }\end{array}$ & $\begin{array}{l}\text { Gregory, 1995; Yegül, } \\
2015 .\end{array}$ \\
\hline & $\begin{array}{l}\text { Lykosou- } \\
\text { ra }\end{array}$ & $\begin{array}{l}\text { Tempio della Despoina: } \\
\text { restauro del naòs, del } \\
\text { pronao e degli anathe- } \\
\text { mata }\end{array}$ & Età adrianea & $\begin{array}{l}\text { IG V,2 520; Billot, 1997, } \\
\text { pp. 279-280. }\end{array}$ \\
\hline 16. & Olimpia & $\begin{array}{l}\text { Terme del Kladeos: nuo- } \\
\text { va erezione }\end{array}$ & Intorno al 100 d.C. & $\begin{array}{l}\text { Mallwitz, 1972, pp. } \\
\text { 274-276; Yegül, 2015, } \\
\text { p. } 252 \text { e fig. } 12.5 .\end{array}$ \\
\hline 17. & Olimpia & $\begin{array}{l}\text { Hospitalia al di sotto } \\
\text { delle terme del Kladeos: } \\
\text { nuova erezione }\end{array}$ & $\begin{array}{l}\text { Dalla metà del II } \\
\text { alla metà del III } \\
\text { secolo d.C. }\end{array}$ & $\begin{array}{l}\text { OlBer VI, in part. } \\
33-37 .\end{array}$ \\
\hline
\end{tabular}




\section{Bibliografia}

Alston, R., van Nijf, O.M. e Williamson, Ch.G. (eds.) (2013). Cults, Creed and Identities in the Greek City after the Classical Age. Leuven, Paris e Walpole MA: Peeters.

Aristodemou, G.A. e Tassios, Th.P. (eds.) (2018). Great Waterworks in Roman Greece. Aqueducts and monumental fountains. Function in context. Oxford: Archaeopress Publishing Ltd.

Aslanidis, K. (2003). The Roman odeion at Epidaurus. JRA, 16, pp. 300-311.

Barresi, P. (2007). Il sofista Flavio Damiano di Efeso e la costruzione di terme-ginnasi nell'Asia Minore romana di età imperiale. In Cordovana e Galli, 2007, pp. 137-151.

Billot, M.F. (1997). Le décor des toits de Grèce du IIe siècle av. au Ier siècle ap. J.C. Traditions, innovations, importations, 1. BCH, 121, pp. 235-290.

Birley, A.R. (1997). Hadrian: the restless emperor. London: Routledge.

Bol, R. (1984). Das Statuenprogramm des Herodes-Atticus-Nymphäums. OlForsch XV. Berlin: de Gruyter.

Bonini, P. (2006). La casa nella Grecia romana. Forme e funzioni dello spazio privato fra I e VI secolo. Antenor Quaderni VI. Padova: Quasar.

Borg, B.E. (ed.) (2004). Paideia: The World of the Second Sophistic. Berlin e New York: de Gruyter.

Bouchon R., Brillet-Dubois P. e Le Meur-Weissman N. (eds.) (2013). Hymnes de la Grèce antique. Approches littéraires et historiques. Actes du colloque international. Lyon, 19-21 juin 2008. Lyon: Maison de l'Orient et de la Méditerranée.

Bressan, M. (2009). Il teatro in Attica e Peloponneso: tra età greca ed età romana: morfologie, politiche edilizie e contesti culturali. Antenor Quaderni XII. Padova: Quasar.

Brown, A.R. (2018). Corinth in Late Antiquity: A Greek, Roman and Christian City. London: I. B. Tauris.

Bukowiecki E., Volpe, R. e Wulf-Rheidt, U. (eds.) (2016). Il laterizio nei cantieri imperiali. Roma e il Mediterraneo. Atti del I Workshop "Laterizio". Roma, 27-28 novembre 2014. Firenze: All' Insegna del Giglio.

Burzachechi, M. (1984). Ricerche epigrafiche sulle antiche biblioteche del mondo greco. RendLinc, 39, pp. 307-338.

Calandra, E. (2007). Plutarco o del restauro. In Cordovana e Galli, 2007, pp. 57-65.

Calandra, E. e Adembri, B. (eds.) (2014). Adriano e la Grecia. Villa Adriana tra classicità ed ellenismo. Studi e ricerche. Verona: Electa.

Camia, F. (2011a). Theoi Sebastoi. Il culto degli imperatori romani in Grecia (provincia Achaia) nel secondo secolo d.C. Meletēmata LXV. Atene: Ethniko Idryma Erevnon.

Camia, F. (2011b). Spending on the agones: the financing of festivals in the cities of Roman Greece. Tyche, 26, pp. 41-76.

Camodeca, G. (2003). L'attività dell'ordo decurionum nelle città della Campania dalla documentazione epigrafica. CahGlotz, 14, pp. 173-186. 
Camporeale, S., Dessales, H. e Pizzo, A. (eds.) (2010). Arqueología de la Construcción II, Los procesos constructivos en el mundo romano: Italia y provincias orientales. Certosa di Pontignano, Siena, 13-15 nov. 2008. Madrid e Merida: Consejo Superior de Investigaciones Científicas.

Caruso, A. (2014). Le biblioteche come centri di cultura nel mondo greco. In Meneghini e Rea, 2014, pp. 61-81.

Clinton, K. (1989). The Eleusinian Mysteries: Roman Initiates and Benefactors, Second Century B.C. to A.D. 267. ANRW II, 18.2, pp. 1499-1539.

Clinton, K. (2005). Eleusis. The inscriptions on stone. Documents of the Sanctuary of the two Goddesses and public documents of the deme. Athens: Archaeological Society at Athens.

Cordovana, O. e Galli, M. (eds.) (2007). Arte e memoria culturale nell'età della Seconda Sofistica. Catania: Edizioni del Prisma.

Dekoulakou, I. (2009). Monumenti delle necropoli di Patrasso durante il dominio romano. In Patrasso colonia di Augusto e le trasformazioni culturali, politiche ed economiche della Provincia di Acaia agli inizi dell'età imperiale romana. Atti del convegno internazionale Patrasso 23-26 Marzo 2006 (pp. 163-210). Tripodes 8. Athene: Scuola Archeologica Italiana di Atene.

DeLaine, J. (1999). Benefactions and Urban Renewal: Bath Buildings in Roman Italy. In DeLaine and Johnston, 1999, pp. 67-74.

DeLaine, J. and Johnston, D.E. (eds.) (1999). Roman Baths and Bathing: Proceedings of the First International Conference on Roman Baths Held at Bath, England, 30 March - 4 April 1992. Portsmouth, RI: Journal of Roman Archaeology.

Di Napoli, V. (2013). Teatri della Grecia romana. Forma, decorazione, funzioni: la provincia d'Acaia. Meletēmata LXVII. Atene: Ethniko Idryma Erevnon.

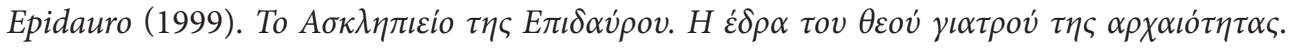
Athens: Periphéreia Peloponnesou.

Fadelli, G. (2018). I viaggi di Adriano. In Lagogianni Georgakarakos e Papi, 2018, p. 33.

Flämig, C. (2007). Grabarchitektur der römischen Kaiserzeit in Griechenland. Rahden: M. Leidorf.

Frei-Stolba, R. y Speidel, M.A. (eds.) (1995). Römische Inschriften. Neufunde, Neulesungen und Neuinterpretationen. Festschrift für Hans Lieb zum 65. Geburtstag dargebracht von seinen Freunden und Kollegen. Basel: Reinhardt.

Galli, M. (2001). Pepaideumenoi am Ort des Heiligen: Kommunikationsformen und euergetische Initiativen in griechischen Heiligtümer zur Zeit des Zweiten Sophistik. In Reusser, 2001, pp. 57-62.

Galli, M. (2002). Die Lebenswelt eines Sophisten. Untersuchungen zu den Bauten und Stiftungen des Herodes Atticus. Mainz a. Rh.: von Zabern.

Galli, M. (2004). 'Creating religious identities': Paideia e religione nella Seconda Sofistica. In Borg, 2004, pp. 315-356.

Galli, M. (ed.) (2013). Roman Power and Greek Sanctuaries. Forms of Interaction and Communication. Tripodes XIV. Athens: Scuola Archeologica Italiana di Atene. 
Gebhard, E.R. (1991). The Isthmian Games and the sanctuary of Poseidon in the early Empire. In Gregory, 1991, pp. 75-94.

Gebhard, E.R. e Gregory, T.E. (eds.) (2015). Bridge of the untiring Sea. The Corinthian Isthmus from Prehistory to late Antiquity. Hesperia Suppl. XLVIII. Athens: The American School of Classical Studies at Athens.

Ginouvès, R. (1972). Le Théâthron a gradins droits et l'Odéon d'Argos. Paris: J. Vrin.

Gogos, S. (1986-1987). Kult und Heiligtümer der Artemis von Aigeira. ÖJh, 57, pp. 109-139.

Goldstein, M.S. (1978). The Settings of the ritual Meal in Greek Sanctuaries: 600-300 B.C., PhD California, Berkeley.

Gregory, T.E. (ed.) (1991). The Corinthia in the Roman Period. JRA suppl. VIII. Ann Arbour: Journal of Roman Archaeology.

Gregory, T.E. (1995). The Roman bath at Isthmia. Preliminary Report 1972-1992. Hesperia, 64, pp. 269-313.

Grosso, F. (1964). La lotta politica ai tempi di Commodo. Torino: Accademia delle Scienze.

Guarducci, M. (1978). Epigrafia greca. IV, Epigrafi sacre pagane e cristiane. Roma: Istituto Poligrafico dello Stato.

Guidoboni, E. (ed.) (1989). I terremoti prima del Mille in Italia e nell'area mediterranea. Bologna: SGA / Istituto Nazionale di Geofisica.

Halfmann, H. (1979). Die Senatoren aus dem östlichen Teil des Imperium Romanum bis sum Ende des 2. Jahrhunderts n. Chr. Hypomnemata LVIII. Göttingen: Vandenhoeck \& Ruprecht.

Halfmann, H. (1986). Itinera principum. Geschichte und Typologie der Kaiserreisen im Römischen Reich. Heidelberger althistorische Beiträge und epigraphische Studien II. Stuttgart: Steiner.

Interdonato, E. (2013). L’Asklepieion di Kos: archeologia del culto. Archeologia Classica Suppl. XII. Roma: "L’Erma" di Bretschneider.

Jost, M. (1985). Sanctuaires et Cultes d’Arcadie. Études péloponnésiennes IX. Paris: J. Vrin.

Kajanto, I. (1982²). The Latin Cognomina. Commentationes Humanarum Litterarum XXXVI.2. Roma: G. Bretschneider.

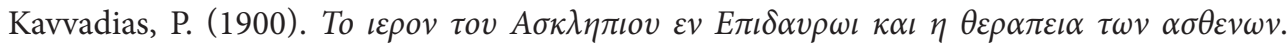

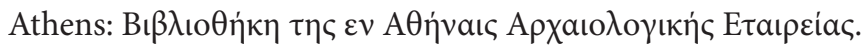

Kraynak, L. (1991). The katagogion at Epidauros. A revised plan. Archaeological News, 16, pp. 1-8.

Lafond, Y. (2006). La mémoire des cités dans le Péloponnèse d'époque romaine (IIe siècle avant J.-C.-IIIe siècle après J.-C.). Collection Histoire. Rennes: Presses Universitaires de Rennes.

Lafond, Y. (2016). Euergetism and religion in the cities of the Peloponnese (first century BC to first century AD) between civic traditions and self-assertion of the elites. In Melfi e Boubou, 2016, pp. 18-26.

Lagogianni Georgakarakos, M. e Papi, E. (eds.) (2018). HADRIANVS - ADPIANOE. O

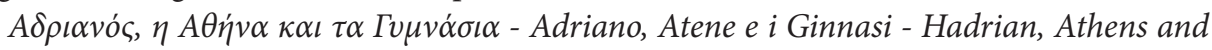


the Gymnasia (mostra Atene 2017-2018). Athens: National Archaeological Museum / Scuola Archeologica Italiana di Atene.

Lolos, Y. (2018). The Hadrianic acqueduct in Corinth. In Aristodemou e Tassios, 2018, pp. 98-108.

Lo Monaco, A. (2009). Il crepuscolo degli dei d'Achaia. Religione e culti in Arcadia, Elide, Laconia e Messenia dalla conquista romana ad età flavia. BCom Suppl. XVII. Roma: "L'Erma" di Bretschneider.

Lo Monaco, A. (2013). Fuori dall'Altis. Tende, bagni e propilei a Olimpia in età ellenistica. In Galli, 2013, pp. 125-142.

Lo Monaco, A. (2014). Adriano in visita agli déi in Grecia. In Calandra e Adembri, 2014, pp. 27-35.

Longfellow, B. (2009). The legacy of Hadrian. Roman monumental civic fountains in Greece. In Kosso, C. e Scott, A. (eds.) (2009). The nature and function of water, baths, bathing, and hygiene from antiquity through the Renaissance. Technology and Change in History XI. Leiden: Brill, pp. 211-232.

Longfellow, B. (2012). Roman Fountains in Greek Sanctuaries. AJA, 116, pp. 133-155.

Luberto, M.R. (2018). Adriano ad Atene. In Lagogianni Georgakarakos e Papi, 2018, p. 32.

Lucore, S.K. e Trümper, M. (eds.) (2013). Greek baths and bathing culture: new discoveries and approaches. Babesch Suppl. XXIII. Leuven: Peeters.

Malacrino, C. (2007). Archelogia e terremoti a Kos. In Malacrino e Sorbo, 2007, pp. 248-273.

Malacrino, C. e Sorbo, E. (eds.) (2007). Architetti, Architettura e città nel Mediterraneo Antico. Milano: B. Mondadori.

Mallwitz, A. (1972). Olympia und seine Bauten. München: Prestel.

Melfi, M. (2007a). I santuari di Asclepio in Grecia. I. Studia Archaeologica CLXII. Roma: L'Erma di Bretschneider.

Melfi, M. (2007b). Asclepio, $\tau \omega \nu \varepsilon v \pi a ı \delta \varepsilon a ~ \eta v \pi \rho o \mu \eta \theta \eta \varsigma$ (Ael. fr. 99 Hercher). Rituale ed evergetismo negli Asklepieia del II sec. d.C. In Cordovana e Galli, 2007, pp. 241-254.

Melfi, M. (2010). Rebuilding the myth of Asklepios at the Sanctuary of Epidauros in the Roman Period. In RomPel III, pp. 329-339.

Melfi, M., Boubou, O. (eds.) (2016). Hellenistic sanctuaries between Greece and Roma, Oxford: Oxford University Press.

Meneghini, R. e Rea, R. (eds.) (2014). La Biblioteca infinita. I luoghi di sapere nel mondo antico (cat. mostra Roma 2014). Milano: Electa.

Meyer, H. (1991). Antinoos. Die archäologischen Denkmäler unter Einbeziehung des numismatischen und epigraphischen Materials sowie der literarischen Nachrichten. Ein Beitrag zur Kunst- und Kulturgeschichte der hadrianisch-frühantoninischen Zeit. München: W. Fink.

Mylonas, G.E. (1961). Eleusis and the eleusinian Mysteries. Princeton: Princeton University Press.

Naso, A. (ed.) (2006). Stranieri e non cittadini nei santuari greci, Convegno Internazionale, Udine, 20-22 nov. 2003. Firenze: Le Monnier. 
Nicolai, R. (1988). Le biblioteche degli Asclepieia. Nuovi Annali per la Scuola Speciale per Archivisti e Bibliotecari, 2, pp. 29-37.

Nielsen, I. (2002). Cultic theatres and ritual drama: a study in regional development and religious interchange between East and West in antiquity. Aarhus Studies in Mediterranean Antiquity IV. Aarhus: Aarhus University Press.

OlBer = Bericht über die Ausgrabungen in Olympia, Berlin: de Gruyter, 1937-.

Oliver, J.H. (1989). Greek Constitutions of Early Roman Emperors from Inscriptions and Papyri. Philadelphia: American Philosophical Society.

Ottati, A. (2016). Costruzione, ricostruzione e 'restaurazione': l'ideologia del principato

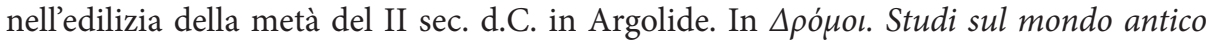
offerti a Emanuele Greco dagli allievi della Scuola Archeologica Italiana di Atene (pp. 239-253). Atene: Pandemos.

Papi, E. (2018). Adriano il Benefattore. In Lagogianni Georgakarakos e Papi, 2018, pp. 46-48. Peek, W. (1969). Inschriften aus dem Asklepieion von Epidauros. Leipzig: Akademie Verlag.

Perilli, L. (2006). "Il Dio ha evidentemente studiato medicina”. Libri di medicina nelle biblioteche antiche. Il caso dei santuari di Asclepio. In Naso, 2006, pp. 472-510.

Piérart, M. (1995). L'Empereur Hadrien et Argos. Une dédicace partiellement inédite d'un temple d'Héra (SEG XI, 340). In Frei-Stolba e Speidel, 1995, pp. 7-16.

Quaß, F. (1993). Die Honoratiorenschicht in den Städten des griechischen Ostens. Untersuchung zur politischen und soziale Entwicklung in hellenistischer und römischer Zeit. Stuttgart: Steiner.

Reusser, C. (ed.) (2001). Griechenland in der Kaiserzeit. Neue Forschungen zur Plastik, Architektur und Topographie. Festschrift zum 60. Geburtstag von D. Willers, Kolloquium Bern 12.-13. Juni 1998. Bern: Institut für Klassische Archäologie der Universität Bern.

Riethmüller, J. (2005). Asklepios: Heiligtümer und Kulte. Studien zu antiken Heiligtümern II. Heidelberg: Archäologie und Geschichte.

Rizakis, A. (ed.) (1996a). Roman Onomastics in the Greek East. Social and Political Aspects. Proceedings of the International Colloquium on Roman Onomastics, Athens 7-9 September 1993. Meletēmata XXI. Athens: Ethniko Idryma Erevnon.

Rizakis, A. (1996b): Anthroponymie et société. Les noms romains dans les provinces hellénophones de l'empire. In Rizakis, 1996a, pp. 11-29.

Rizakis, A. (2007). Urban elites in the Roman East. Enhancing Regional Position and Social Superiority. In Rüpke, 2007, pp. 317-330.

Rizakis, A. (2009). Supra-civic landowning and supra-civic euergetic activities of urban elites in the imperial Peloponnese. In Being Peloponnesian. Cohesion and diversity through time. International Conference, University of Nottingham, 31 March-1 April 2007. On line.

Rizakis, A. (2015). The Greek Ruling Class under the Empire: a Privileged Mediator between Rome and the Cities. Kodai, 16, pp. 143-159.

Rizakis, A.D., Camia, F. e Zoumbaki, S. (eds.) (2017). Social Dynamics under Roman Rule. Mobility and Status Change in the Provinces of Achaia and Macedonia. Proceedings of 
a Conference Held at the French School of Athens, 30-31 May 2014. Meletēmata LXXIV. Athens: Ethniko Idryma Erevnon.

Rizakis, A.D. e Zoumbaki, S. (2017). Local elites and social mobility in Greece under the Empire: the Cases of Athens And Sparta. In Rizakis, Camia e Zoumbaki, 2017, pp. 159-180.

Robert, L. (1937). Études Anatoliennes. Recherches sur les inscriptions Grecques de l'Asie Mineure. Paris: de Boccard.

Rogers, D.K. (2018). Shifting ideas: approaches to the public water-displays of Roman Greece. In Aristodemou e Tassios, 2018, pp. 173-192.

RomPel I = Rizakis, A.D. e Zoumbaki, S. (eds.) (2001). Roman Peloponnese I. Roman Personal Names in their social Context. Meletēmata XXXI. Athens: Ethniko Idryma Erevnon.

RomPel II = Rizakis, A.D., Zoumbaki, S. e Lepenioti, Cl. (eds.) (2004). Roman Peloponnese II. Roman Personal Names in their social Context. Meletēmata XXXVI. Athens: Ethniko Idryma Erevnon.

RomPel III = Rizakis, A.D. e Lepenioti, Cl. (eds.) (2010). Roman Peloponnese III. Society, Economy and Culture under the Roman Empire: Continuity and Innovation. Meletēmata LXIII. Athens: Ethniko Idryma Erevnon.

Roy, J. (2016). Pausanias and Hadrian, Mantinea and Bithynion. Histos, 10, pp. 111-131.

Rüpke, J. (2007). A Companion to Roman Religion. Malden MA: Blackwell.

Schmidt-Dounas, B. (2000). Geschenke erhalten die Freundschaft. Politik und Selbstdarstellung im Spiegel der Monumente. Berlin: Akademie Verlag.

Schörner, G. (2000). Stiftungen von Badeanlagen im hellenistischen und kaiserzeitlichen Griechenland. In Cialowicz, K.M. e Ostrowski J.A. (eds.) (2000). Les civilisations du bassin méditerranéen, Hommages à Joachim Śliwa. Cracovie: Institut d’Archéologie, pp. 307-315.

Schörner, G. (2003). Votive im römischen Griechenland. Untersuchungen zur späthellenistischen und kaiserzeitlichen Kunst- und Religionsgeschichte. Stuttgart: Steiner.

Schörner, G. (2013). Officials as dedicators in post-classical poleis. In Alston, van Nijf e Williamson, 2013, pp. 197-213.

Settis, S. (1968). Il ninfeo di Erode Attico a Olympia e il problema della composizione della Periegesi di Pausania. AnnPisa, 2.37, pp. 1-63.

Sinn, U., Ladstätter, G. e Martin, A. (1993). Bericht über das Forschungsprojekt 'Olympia während der römischen Kaiserzeit' II. Die Arbeiten im Jahr 1993. Nikephoros, 6, pp. 153-158.

Sinn, U., Ladstätter, G. e Martin, A. (1996). Bericht über das Forschungsprojekt 'Olympia während der römischen Kaiserzeit und in der Spätantike’ V. Die Arbeiten im Jahr 1995, Teil 2. Nikephoros, 9, pp. 199-228.

Specht, S. (2004). Ein Stück Rom in Griechenland: der kaiserzeitliche Umbau des Leonidaion in Olympia. In Reusser, 2004, pp. 33-41.

Tassios, T.P. (2018). Roman Aqueducts in Greece. In Aristodemou e Tassios, 2018, pp. 1-9. 
Torelli, M. (2010). P. Licinius Priscus Iuventianus e la ricostruzione antonina del Palaimonion di Istmia. In Dialéctica histórica y compromiso social. Homenaje Domingo Plácido (pp. 555-582). Madrid: Libros Pórtico.

Trümper, M. (2013). Urban Context of Greek Baths. In Lucore e Trümper, 2013, pp. 33-72.

Trümper, M. (2014). Bathing in the sanctuaries of Asklepios and Apollo Maleatas at Epidauros. In Approaching the ancient artifact. Representation, narrative, and function. A Festschrift in honor of H. Alan Shapiro (pp. 211-231). Berlin: de Gruyter.

Vitti, P. (2010). Regola ed eccezione nei cantieri romani della provincia Acaia. In Camporeale, Dessales e Pizzo, 2010, pp. 301-326.

Vitti, P. (2016). Building Roman Greece. Innovation in vaulted construction in the Peloponnese. Studia Archaeologica CCVI. Roma: "L'Erma" di Bretschneider.

Vitti, P. (2016). La costruzione nel Peloponneso romano: tradizione, sperimentazione e innovazione nell' uso del laterizio", in Bukowiecki, Volpe e Wulf-Rheidt, 2016, pp. 190-205.

Vitti, M. e Vitti, P. (2010). Trasmissione ed adattamento delle tecniche costruttive romane in Peloponneso: il caso di Trezene. In RomPel III, pp. 267-290.

Wagman, R. (1995). Inni di Epidauro. Biblioteca di Studi antichi LXXV. Pisa: Giardini editori.

Wagman, R. (2013). From song to monument: sacred poetry and religious revival in Roman Epidaurus. In Bouchon, Brillet-Dubois e Le Meur-Weissman, 2013, pp. 219-231.

Waldstein, C. (1902). The Argive Heraeum, I. Boston-New York: Houghton, Mifflin and Company.

Wassenhoven, M-E. (2012). The Bath in Greece in Classical Antiquity. The Peloponnese. BAR 2368. Oxford: Archaeopress.

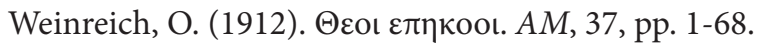

Yegül, F.K. (1991). The Roman baths at Isthmia in their Mediterranean context. In Gregory, 1991, pp. 95-112.

Yegül, F.K. (2015). Roman bath at Isthmia and Sanctuary baths in Greece. In Gebhard e Gregory, 2015, pp. 247-268.

Zoumbaki, S. (2008). The Composition of the Peloponnesian Elites in the Roman period and

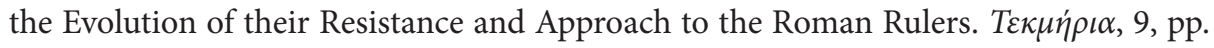
25-51.

Zoumbaki, S. (2010). Elean relations with Rome and the Achaean Koinon and the role of Olympia. In RomPel III, pp. 111-127.

Zunino, M.L. (1997). Hiera Messeniaka. La storia religiosa della Messenia dall'età micenea all'età ellenistica. Udine: Forum. 